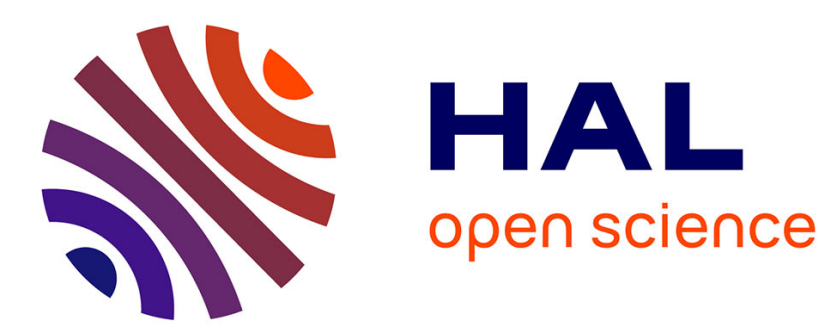

\title{
Electric field measurements in plasmas: how focusing strongly distorts the E-FISH signal
}

Tat Loon Chng, Svetlana Starikovskaia, Marie-Claire Schanne-Klein

\section{To cite this version:}

Tat Loon Chng, Svetlana Starikovskaia, Marie-Claire Schanne-Klein. Electric field measurements in plasmas: how focusing strongly distorts the E-FISH signal. Plasma Sources Science and Technology, 2020, 29 (12), pp.125002. 10.1088/1361-6595/abbf93 . hal-03070299

\section{HAL Id: hal-03070299 \\ https://hal.science/hal-03070299}

Submitted on 6 Jan 2021

HAL is a multi-disciplinary open access archive for the deposit and dissemination of scientific research documents, whether they are published or not. The documents may come from teaching and research institutions in France or abroad, or from public or private research centers.
L'archive ouverte pluridisciplinaire $\mathbf{H A L}$, est destinée au dépôt et à la diffusion de documents scientifiques de niveau recherche, publiés ou non, émanant des établissements d'enseignement et de recherche français ou étrangers, des laboratoires publics ou privés. 


\title{
Electric Field Measurements in Plasmas: How Focusing Strongly Distorts the E-FISH Signal
}

\author{
Tat Loon Chng ${ }^{1}$, Svetlana M. Starikovskaia ${ }^{1}$, Marie-Claire Schanne-Klein ${ }^{2}$ \\ ${ }^{1}$ Laboratoire de Physique des Plasmas, (CNRS, École Polytechnique, Sorbonne Universités, Univ. \\ Paris-Sud), Institut Polytechnique de Paris, F-91128, Palaiseau, France \\ ${ }^{2}$ Laboratoire d'Optique et Biosciences (LOB), École Polytechnique, CNRS, Inserm, Institut \\ Polytechnique de Paris, F-91128, Palaiseau, France
}

\begin{abstract}
Electric field induced second harmonic generation (E-FISH) has recently demonstrated significant potential as a method for making absolute electric field measurements in non-equilibrium plasmas and gas discharges. Previous studies have relied on the plane-wave approximation in quantifying these measurements, while in reality, focused laser beams are almost always used. In this work, we perform a theoretical and experimental study using focused Gaussian beams, and examine the consequent effects on the E-FISH signal. We show that in addition to important parameters such as the external electric field strength, wave vector mismatch and Rayleigh range, the signal is strongly influenced by the full length and shape of this external field profile. We attribute this to the Gouy phase shift associated with focused beams, and note that analogous effects have been previously observed in second and third harmonic generation microscopy. This dependence of the E-FISH signal on the spatial profile of the external field is worth highlighting since it is often not easily determined a priori in a plasma, and neglecting its influence could lead to an incorrect electric field measurement. To minimize any inaccuracies associated with this issue, we propose several recommendations to consider when using the E-FISH diagnostic with focused beams.
\end{abstract}




\section{Introduction}

Electric field induced second harmonic generation or E-FISH, is a non-linear optical phenomenon, which has lately been redeveloped and successfully employed as a diagnostic for electric field measurements in plasmas [1,2]. The utility of this technique, particularly for nonequilibrium plasma applications, has been illustrated by its use over a wide spectrum of studies, from nanosecond pulse discharges for understanding fast ionization wave development $[3,4]$, surface dielectric barrier discharges [5] and atmospheric pressure plasma jets [6], to DC corona discharges [7]. The main reasons for this popularity are its overall simplicity, as well as the ability to achieve excellent measurement resolution in both time (sub-ns) and space (sub-mm) [1]. Most recent developments include the probing of 1-D electric fields [6,8 \& 9] by line focusing with a cylindrical lens, as well as obtaining simultaneous electric field vector information through the use of a polarizing beamsplitter on the detection end [10].

A common aspect of the foregoing studies is the reliance on a plane-wave approximation - given by eqn. (1) in section 2.1 - to relate the measured signal to the externally applied electric field of interest. Additionally, this mathematical description is often applied with an attendant assumption that the signal originates predominantly from the focal region of the probe beam, rather than the entire overlap extent between the laser and the external field. The premise for this assumption is the quadratic dependence of the second harmonic generation (SHG) on the probe beam intensity, which reasons that most of the non-linear interaction, and thus signal, originates from the confocal region of the probe beam (i.e. where the laser intensity is the strongest). While such an assumption seems plausible, it is also somewhat imperfect, since a plane wave by definition implies a confocal parameter (twice the Rayleigh range) of infinity. Yet this assumption is also crucial for accurate implementation of the diagnostic, since it allows the signal measured from a known external field to be used as the basis for calibration in a plasma, without the need for any explicit consideration or information on the length scales (of the external field profiles) of both systems. In other words, the confocal parameter is assumed to define the spatial resolution of the measurement.

In this paper, we further assess the validity of this important assumption by specifically examining whether (or how) the spatial profile of the externally applied field, in addition to other parameters such as the Rayleigh range, affects the E-FISH signal. A focused Gaussian beam analysis, supported by relevant experiments, is used to achieve a more accurate description of the E-FISH signal. In the broader picture, we seek a deeper understanding of the strengths and limitations of the E-FISH diagnostic as a method for electric field measurements. A key finding of this work is

that the full length and shape of the external field profile should be considered as a separate parameter which influences the resulting signal. This poses a challenge for obtaining accurate, absolute electric field data, an issue we address in the closing stages of the paper. 


\section{Theoretical analysis}

\subsection{Plane-wave approximation}

(a)

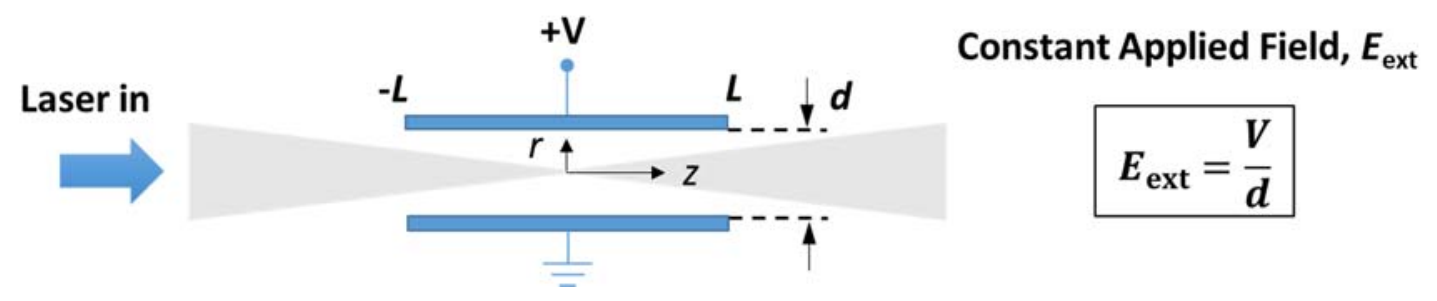

(b)

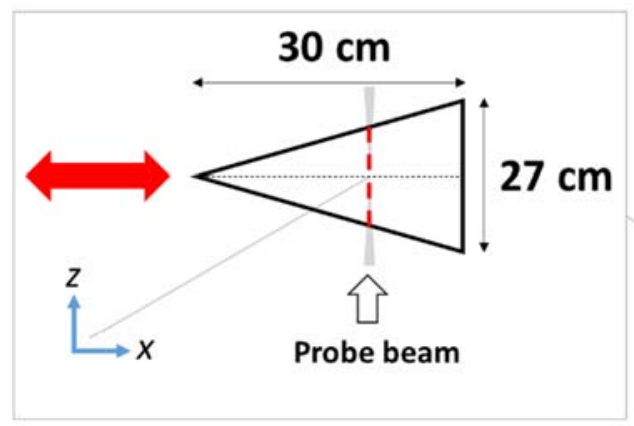

Probe beam

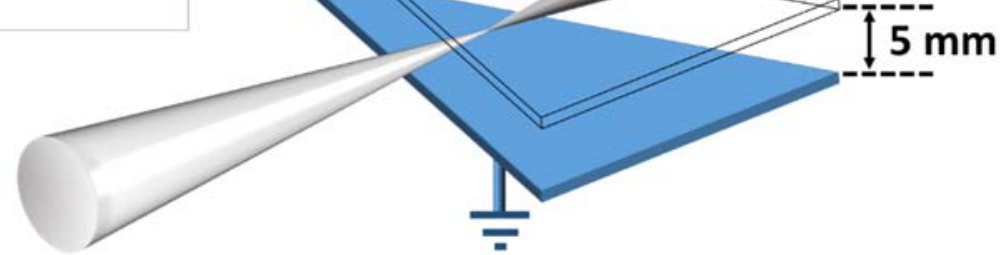

Figure 1. (a) Schematic of a canonical geometry typically used in E-FISH diagnosis. The top electrode is held at a potential of $V$ with respect to the grounded bottom electrode. (While this illustration presents a focused probe beam, it should be understood that in the case of a planewave, the intensity of the probe beam is constant with respect to space and time.) Corresponding schematic of the triangular-shaped, parallel-plate electrodes used for the experiments. By translating the electrodes (red arrow in figure inset), the effective electrode length (vertical red dashed line) seen by the E-FISH probe beam is varied.

We begin by describing the E-FISH signal in terms of a canonical electrode geometry comprising two parallel plates, each of length $2 L$, and separated by an interelectrode gap $d$, as shown in figure 1(a). For such a geometry, the resulting electric field $E_{\text {ext }}$, may be assumed uniform, if edge (or fringing) effects near the electrode edges are neglected. In the plane-wave approximation, the intensity of the second harmonic signal, $I^{(2 \omega)}$ for this configuration is given by:

$$
I_{i}^{(2 \omega)} \propto\left[\alpha_{i j k l}^{(3)} \cdot N \cdot\left(E_{\mathrm{ext}}\right)_{j} \cdot E_{k}{ }^{(\omega)} E_{l}{ }^{(\omega)}\right]^{2} \cdot\left|\int_{-L}^{L} \exp (\mathrm{i} \cdot \Delta k z) \mathrm{d} z\right|^{2}
$$




$$
=\left[\alpha_{i j k l}^{(3)} \cdot N \cdot\left(E_{\mathrm{ext}}\right)_{j} \cdot E_{k}{ }^{(\omega)} E_{l}{ }^{(\omega)} \cdot \frac{\sin (\Delta k \cdot L)}{\Delta k}\right]^{2}
$$

where $\alpha_{i j k l}^{(3)}$ is the third order, non-linear hyperpolarizability (a material-dependent, $4^{\text {th }}$ order tensor), $N$ is the number density of particles participating in the non-linear interaction (usually assumed to be the neutral gas density), $E_{\text {ext }}$ is the externally applied electric field (i.e. of the plasma), $E^{(\omega)}$ is the electric field of the laser beam at the fundamental frequency $\omega, \Delta k$ is the wave vector mismatch (i.e. difference between the fundamental and second harmonic wave vectors), and $2 L$, the electrode length, effectively defines the interaction length, $L_{\text {int }}$, or region of overlap between $E_{\text {ext }}$ and $E^{(\omega)}$. (Here, z denotes the beam propagation axis.)

Eqn. (1) is valid when the (second harmonic) conversion efficiency is very weak, and negligible depletion of the probe beam (i.e. $I^{(\omega)}$ remains constant throughout the interaction) can thus be safely assumed. In addition, it implicitly assumes a centrosymmetric medium (i.e. in the absence of $E_{\text {ext }}$, no SHG from lower order processes), an assumption which can be easily verified via experiment, or enforced in terms of an appropriate choice of gas composition. Furthermore, one notes that in a gas or plasma, the wave vector mismatch, $\Delta k$ (effectively proportional to the difference in the indices of refraction at the fundamental, $n^{(\omega)}$ and second harmonic frequencies, $n^{(2 \omega)}$ ), is necessarily negative (i.e. $\Delta k \propto\left[n^{(\omega)}-n^{(2 \omega)}\right]<0$ ). The effect of electrode length on the E-FISH signal in the plane-wave approximation is shown in figure 2 for $\Delta k=-0.5 \mathrm{~cm}^{-1}$. Analogous to SHG from non-centrosymmetric media, constructive interference between the fundamental and second harmonic waves leads to an increase in signal with electrode length up to $L_{\text {coh }}=\frac{\pi}{|\Delta k|}=6.28 \mathrm{~cm}$, beyond which they begin to destructively interfere, and the power in the second harmonic gradually flows back to the fundamental. The sine-squared term in eqn. (1) ensures that this interaction repeats itself with a period equal to $2 L_{\mathrm{coh}}=\frac{2 \pi}{|\Delta k|}=12.56 \mathrm{~cm}$. This implies that, in the plane-wave approximation, the E-FISH signal attains a maximum for electrode lengths which are multiples of $(2 p+1) \cdot L_{\text {coh }}$ where $p$ is a positive integer. 


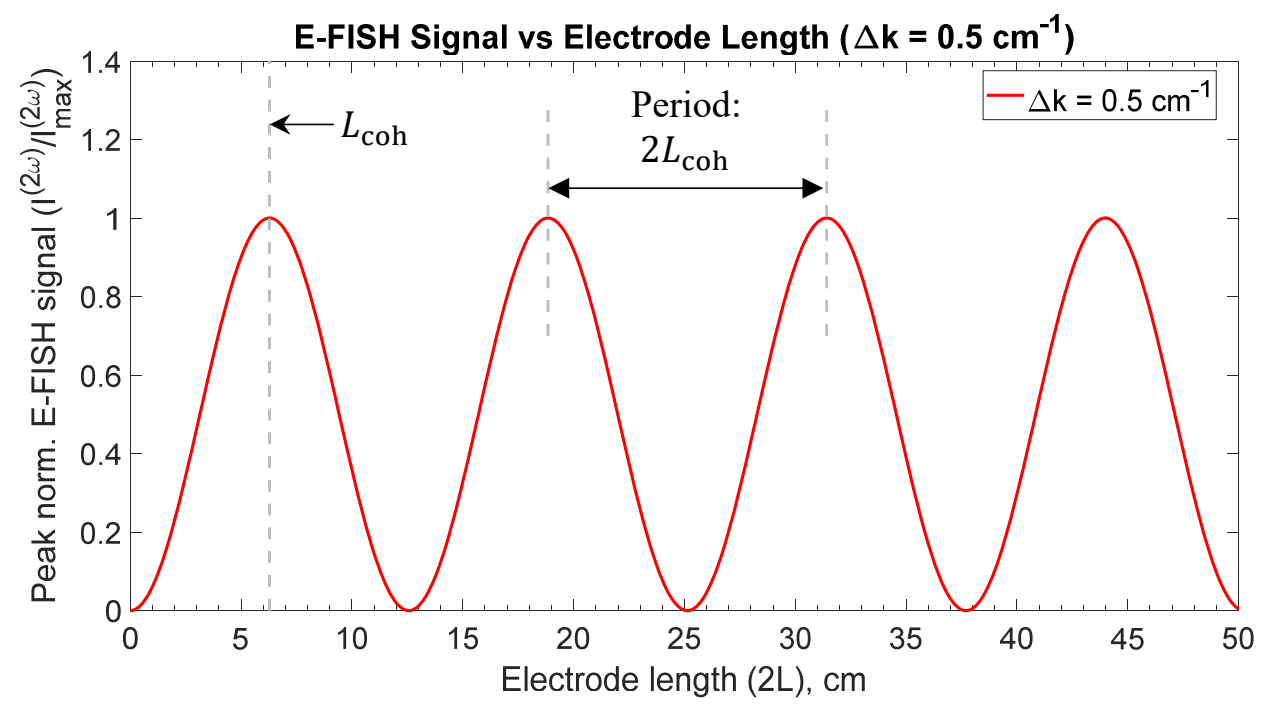

Figure 2. Effect of electrode length, $2 L$ on the peak-normalized E-FISH signal in the plane-wave approximation given by eqn. (1) for $\Delta k=-0.5 \mathrm{~cm}^{-1}$.

A point of essence to this work, is the fact that in previous studies involving E-FISH as an electric field diagnostic, the interaction length, $L_{\text {int }}$, has often been defined as the confocal parameter, or twice the Rayleigh range, $2 z_{R}$, of the laser beam (i.e. $L_{\text {int }}=2 z_{R}$ ). To illustrate this point, we adopt a modified description of the plane-wave approximation, by considering the case of a probe beam of varying width, and therefore intensity, along the $z$-axis. The width variation is similar to the one of Gaussian beams: $w^{(\omega)}(z)=w_{0}^{(\omega)} \sqrt{\left[1+\left(\frac{z}{z_{R}}\right)^{2}\right]}$ where $w^{(\omega)}(z)$ is the Gaussian beam width (or radius), $w_{o}^{(\omega)}$ is the beam waist (i.e. beam width at $z=0$ ) and $z_{R}$ is the Rayleigh range of the probe beam. (Note that for simplicity, we neglect all variations in the radial direction.) Mathematically, this may be implemented by expressing the electric field of the probe beam as follows:

$$
E^{(\omega)}(z)=E_{o}^{(\omega)} \cdot\left[\frac{w^{(\omega)}(z)}{w_{o}^{(\omega)}}\right]=\frac{E_{o}^{(\omega)}}{\sqrt{\left[1+\left(\frac{z}{z_{R}}\right)^{2}\right]}}
$$

where $E_{o}{ }^{(\omega)}$ is the amplitude of the electric field of the probe beam (at the fundamental frequency $\omega$ ). By substituting eqn. (2) into eqn. (1), we obtain a modified expression for the E-FISH intensity, given by:

$$
I_{i}^{(2 \omega)} \propto\left[\alpha_{i j k l}^{(3)} \cdot N \cdot\left(E_{\text {ext }}\right)_{j} \cdot\left(E_{o}^{(\omega)}\right)_{k} \cdot\left(E_{o}^{(\omega)}\right)_{l}\right]^{2} \cdot\left|\int_{-L}^{L} \frac{\exp (\mathrm{i} \cdot \Delta k z)}{\left[1+\left(\frac{Z}{z_{R}}\right)^{2}\right]} \mathrm{d} z\right|^{2}
$$

This modified expression for the E-FISH intensity provides a basis for evaluating the effect of electrode length on the E-FISH signal, within the framework of the plane-wave approximation. 
Noting further that $E_{o}^{(\omega)}$ and all other terms in the square brackets of eqn. (3) are typically invariant with respect to the beam propagation $(z)$ axis, the effect of electrode length on the E-FISH intensity is fully captured by the expression:

$$
\Lambda=\left|\int_{-L}^{L} \frac{\exp (\mathrm{i} \cdot \Delta k z)}{\left[1+\left(\frac{Z}{z_{R}}\right)^{2}\right]} \mathrm{d} z\right|^{2}
$$

Figure 3(a) plots $\Lambda$ as a function of electrode length, $2 L$, for three different values of the Rayleigh range, namely $z_{R}=0.847 \mathrm{~mm}, 3.39 \mathrm{~mm}$ and $9.41 \mathrm{~mm}$. These values of $z_{R}$ correspond to estimates for three different commonly used focal length lenses (f $=15 \mathrm{~cm}, 30 \mathrm{~cm}$ and $50 \mathrm{~cm}$ respectively), based on an initial (unfocused) beam waist of $3 \mathrm{~mm}$, and a probe laser wavelength of $1064 \mathrm{~nm}$. The wave vector mismatch, $\Delta k$ is assigned a value of $-0.5 \mathrm{~cm}^{-1}$ following our previous work [11], and corresponds respectively to fundamental and second harmonic wavelengths of $1064 \mathrm{~nm}$ and $532 \mathrm{~nm}$ in air, with the indices of refraction calculated at room temperature and pressure [12]. These chosen values are retained and re-used consistently within this study, and have been selected to match the conditions of the experiments presented in section 3.1. All three curves in figure 3(a) clearly flatten out at large values of $2 L$, an indication that the E-FISH signal becomes independent of electrode length as $L$ increases. As mentioned earlier, this behavior may be understood in terms of the idea that the signal originates predominantly from within the focal region (i.e. $L_{\text {int }}$ ) due to the higher laser intensity, and is therefore unaffected by any changes in the far field. In addition, the signal also increases with $z_{R}$, in line with the argument that elongating the focal region leads to a stronger signal due to a longer buildup length.

To further examine the spatial origin of the signal, we track the spatial buildup (or evolution) of the E-FISH intensity by evaluating the expression:

$$
\Lambda(z)=\left|\int_{-L}^{z} \frac{\exp (\mathrm{i} \cdot \Delta k z)}{\left[1+\left(\frac{z}{z_{R}}\right)^{2}\right]} \mathrm{d} z\right|^{2} \text { for }-L \leq z \leq L .
$$

We remark that a plot of $\Lambda$ versus $z$ based on eqn. (5) is equivalent to following the variation of the E-FISH signal along the $z$-axis, although in an experiment, what is measured is only the final signal $(\equiv \Lambda)$ at $z=L$. Figure $3(\mathrm{~b})$ shows such a plot for a selected value of $L=6.4 \mathrm{~cm}$ (or electrode length of $12.8 \mathrm{~cm}$ ). Indeed, about $60 \%$ of the final signal is attained within one confocal parameter, and close to the entire signal is achieved within 5 confocal parameters (given respectively by gray and blue dashed lines). 


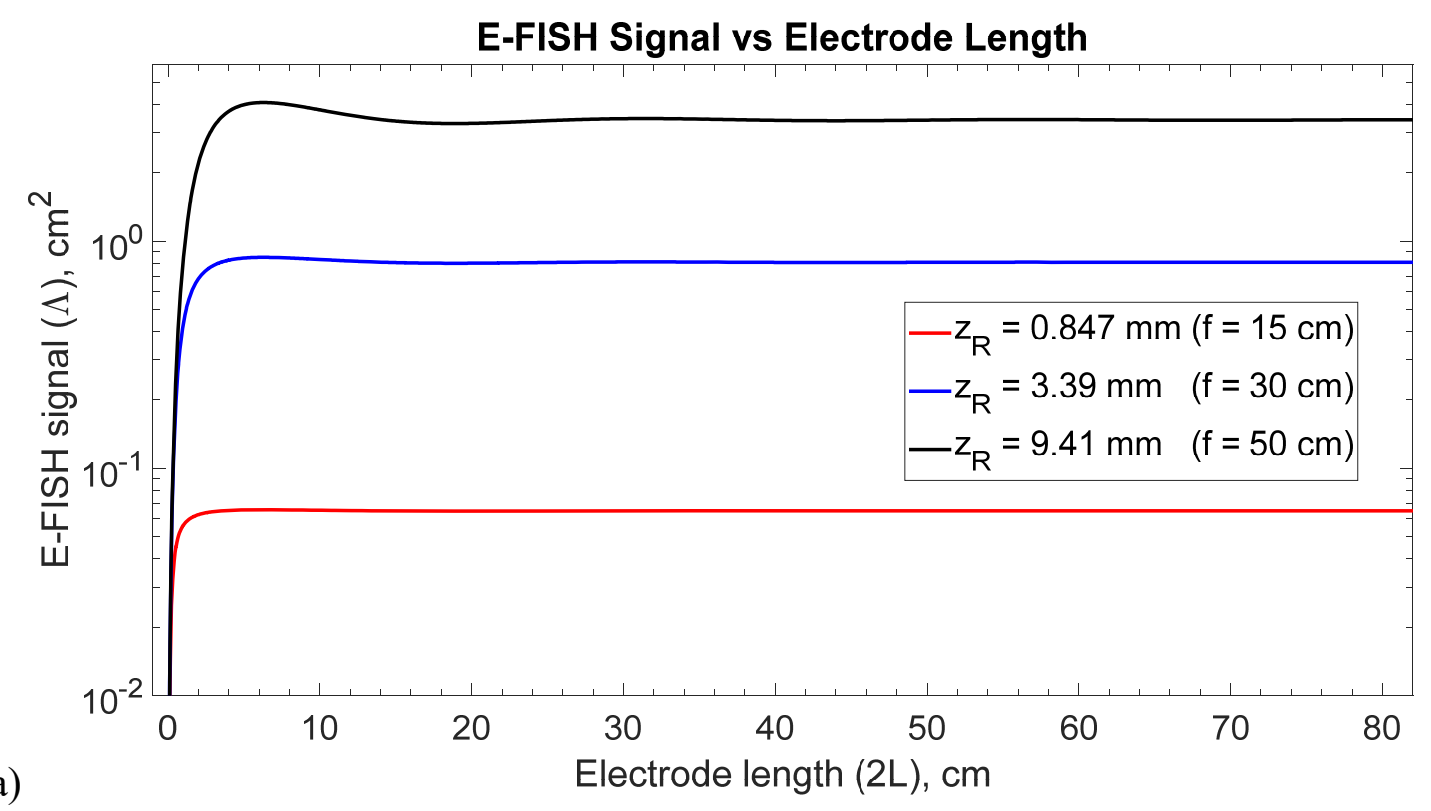

(a)

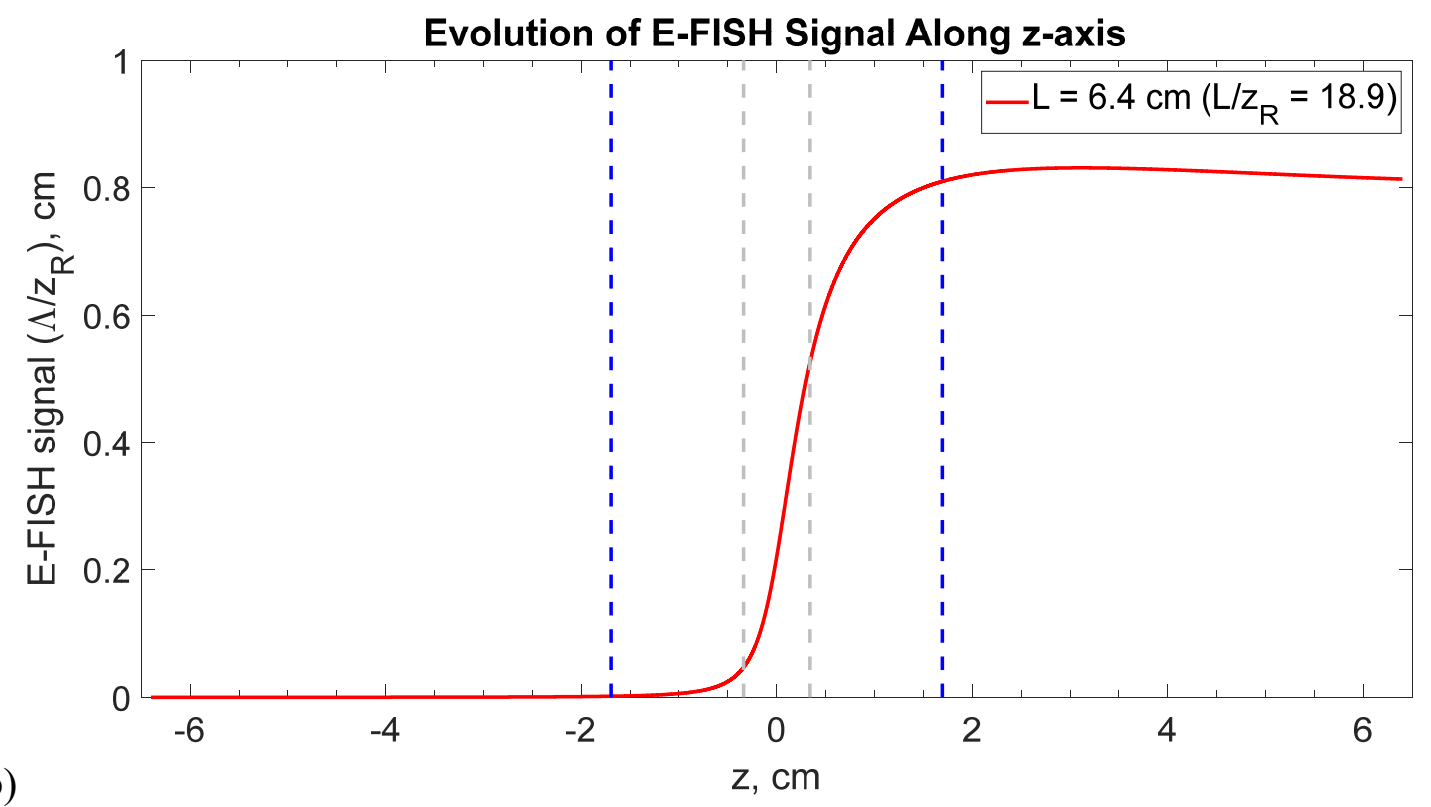

Figure 3. E-FISH signal in the modified plane-wave approximation. (a) Effect of electrode length, $2 L$ on $\Lambda$ (based on eqn. (4)) for three different $z_{R}$ and $\Delta k=-0.5 \mathrm{~cm}^{-1}$ (note the logarithmic scale on the vertical axis). (b) Spatial (z) evolution of the E-FISH signal (given by eqn. (5)) in a constant external field, for $L=6.4 \mathrm{~cm}$, and $z_{R}=3.39 \mathrm{~mm}$. Gray and blue vertical dashed lines correspond to $z= \pm z_{R}$ and $\pm 5 z_{R}$ respectively.

\subsection{Governing equations for a focused Gaussian beam}

While eqn. (3) (and the results that follow) provide an approximate approach to describing the E-FISH signal for a focused beam, a fundamental limitation is that it neglects all phase variations 
in the electric field, which are often extremely important in non-linear interactions. For an accurate description of the E-FISH process, the complex amplitude of the electric field for a focused probe beam should be written in complex notation (for a Gaussian beam) as [13]:

$$
E^{(\omega)}=\frac{E_{o}^{(\omega)}}{\left[1+i \cdot\left(\frac{Z}{z_{R}}\right)\right]} \cdot \exp \left\{\frac{-r^{2}}{\left[w_{o}^{(\omega)}\right]^{2} \cdot\left[1+i \cdot\left(\frac{Z}{z_{R}}\right)\right]}\right\}
$$

where $r$ is the radial (or transverse) axis and $z$ is the direction of propagation of the Gaussian beam. Eqn. (6) is the rigorous solution of the paraxial wave equation and accounts for not only the radial and longitudinal variations in the electric field intensity, but also their respective phase variations. Following [13], by substituting eqn. (6) into the non-linear wave equation, one gets an expression for the E-FISH intensity for a focused Gaussian beam. For the canonical electrode geometry shown in figure $1(\mathrm{a})$, this expression (at $r=0$ ) is given as:

$$
I_{i}^{(2 \omega)} \propto\left[\alpha_{i j k l}^{(3)} \cdot N \cdot\left(E_{\mathrm{ext}}\right)_{j} \cdot\left(E_{o}^{(\omega)}\right)_{k}\left(E_{o}^{(\omega)}\right)_{l}\right]^{2}\left|\int_{-L}^{L} \frac{\exp (\mathrm{i} \cdot \Delta k z)}{\left[1+\mathrm{i} \cdot\left(\frac{Z}{Z_{R}}\right)\right]} \mathrm{d} z\right|^{2}
$$

where the origin is located at mid-height and mid-length of the two electrodes. It should be noted that the expression within the modulus on the r.h.s. of eqn. (7) is identical to that for SHG from a non-centrosymmetric medium (of length $2 L$ ) if one assumes that $E_{\text {ext }}$ is constant. However, a zvarying externally applied field may be readily evaluated without any loss of accuracy by considering $E_{\text {ext }}(z)$ as part of the integrand.

An important distinction between the analysis for a focused beam and plane wave, is the variation in the E-FISH intensity along the radial axis. To account for both the $r$ and $z$ intensity variations in the case of focused beams, it is therefore more appropriate to describe the E-FISH signal in terms of a power. As further discussed in [13], in arriving at eqn. (7), the second harmonic EFISH light has been written as a Gaussian beam possessing the same Rayleigh range as that of the fundamental. The resulting second harmonic E-FISH power, $P^{(2 \omega)}$ can thus be expressed as:

$$
P^{(2 \omega)} \propto\left[w_{o}^{(2 \omega)}\right]^{2} \cdot I_{i}^{(2 \omega)}
$$

where $w_{o}^{(2 \omega)}$ is the Gaussian beam waist at the second harmonic frequency, $2 \omega$, and eqn. (8) is in fact a common expression for the power of a Gaussian beam. Hence, we may also write an equivalent expression for the power carried by the fundamental frequency:

$$
P_{o}^{(\omega)} \propto\left[w_{o}^{(\omega)}\right]^{2} \cdot I_{o}^{(\omega)}
$$

where $I_{o}^{(\omega)}$ is the peak intensity and $I_{o}^{(\omega)} \propto\left|E_{o}^{(\omega)}\right|^{2}$. An important difference between eqns. (8) and (9) is that while the power contained in the probe beam is necessarily constant (assuming negligible depletion), the E-FISH power is in fact a function of $z$. 
By substituting eqns. (8) and (9) into eqn. (7), we get:

$$
P^{(2 \omega)} \propto\left[\alpha_{i j k l}^{(3)} \cdot N \cdot E_{\mathrm{ext}} \cdot P_{o}^{(\omega)}\right]^{2} \cdot \frac{\left[w_{o}^{(2 \omega)}\right]^{2}}{\left[w_{o}^{(\omega)}\right]^{4}} \cdot\left|\int_{-L}^{L} \frac{\exp (\mathrm{i} \cdot \Delta k z)}{\left[1+\mathrm{i} \cdot\left(\frac{z}{z_{R}}\right)\right]} \mathrm{d} z\right|^{2}
$$

Adopting the usual definition of the Rayleigh range in terms of the beam waist,

$$
z_{R}=\frac{\pi w_{o}^{2}}{\lambda}
$$

where $\lambda$ is the laser wavelength, and noting further that the beam waists of the fundamental and second harmonic waves differ only by a constant prefactor (i.e. $w_{o}{ }^{(\omega)}=\sqrt{2} w_{o}{ }^{(2 \omega)}$ ) since they share the same Rayleigh range, eqn. (10) may be simplified as:

$$
P^{(2 \omega)} \propto\left[\alpha_{i j k l}^{(3)} \cdot N \cdot E_{\text {ext }} \cdot P_{o}^{(\omega)}\right]^{2} \cdot\left(\frac{1}{z_{R}}\right) \cdot\left|\int_{-L}^{L} \frac{\exp (\mathrm{i} \cdot \Delta k z)}{\left[1+\mathrm{i} \cdot\left(\frac{Z}{z_{R}}\right)\right]} \mathrm{d} z\right|^{2} .
$$

In a manner similar to the derivation of eqn. (4), inspection of eqn. (12) reveals that if the terms in the square brackets may be held constant, the dependence of the E-FISH power on the length of the external field profile is contained only within the expression:

$$
P^{(2 \omega)} \propto\left(\frac{\Lambda^{\prime}}{z_{R}}\right) ; \text { where } \Lambda^{\prime}=\left|\int_{-L}^{L} \frac{\exp (\mathrm{i} \cdot \Delta k z)}{\left[1+\mathrm{i} \cdot\left(\frac{z}{z_{R}}\right)\right]} \mathrm{d} z\right|^{2} .
$$

The $\left(\frac{1}{z_{R}}\right)$ term in eqns. (12) and (13) essentially accounts for the intensity variation in the probe beam as a function of the focusing, for a constant input power, $P_{o}^{(\omega)}$. More importantly, it is worth pointing out that the integrand in the above expression differs from the plane-wave approximation (see eqn. (1)) by the factor $\left[1+\mathrm{i} \cdot\left(\frac{z}{z_{R}}\right)\right]$ in the denominator. (In fact, we recover the expression for the plane wave as $z_{R} \gg L$.) As pointed out in [14], this factor can be expanded into its less compact, but more illuminating form $\frac{\exp \left[-\mathrm{i} \cdot \operatorname{atan}\left(\frac{z}{z_{R}}\right)\right]}{\sqrt{1+\left(\frac{z}{z_{R}}\right)^{2}}}$, where the numerator contains an expression which corresponds to the Gouy phase $\operatorname{shift} \phi_{\mathrm{g}}(z)=+\operatorname{atan}\left(\frac{z}{z_{R}}\right)[15]$ and the denominator is related to the z-evolution of the Gaussian beam width (see eqn. (2)).

Interestingly, one finds that the integral within the modulus of eqn. (13) approaches zero, as $L$ approaches infinity. This is analogous to the well-known result that SHG or THG from a noncentrosymmetric medium (with negative phase mismatch) approaches zero for focused Gaussian beams in an infinite medium [13]. (However, $\Lambda^{\prime}$ remains finite provided that $L<\infty$.) From the perspective of the E-FISH diagnostic, this suggests that the measured signal has an explicit 
dependence on the spatial extent of the external field (i.e. electrode length $2 L$ in fig. 1), in addition to other parameters such as the magnitude of the external field, $E_{\text {ext }}$, wave vector mismatch $\Delta k$, and the choice of $z_{R}$. More crucially, this runs contrary to the earlier discussed idea of designating the confocal parameter as the interaction length, $L_{\text {int }}$. Rather, it implies that $L_{\text {int }}$ should instead be treated as the entire length of the external field profile, which in this case corresponds to the electrode length, $2 L$.

Figure 4(a) plots $\frac{\Lambda^{\prime}}{z_{R}}$ as a function of the electrode length, $2 L$ for the same values of $z_{R}$ and $\Delta k$ as in figure 3(a). In agreement with the hypothesis discussed above, figure 4(a) predicts that $\frac{\Lambda^{\prime}}{z_{R}}$ (taken henceforth to be synonymous with the E-FISH signal) exhibits a strong and periodic dependence on the electrode length. Furthermore, with the exception of the first peak (a point we address in section 2.3), successive peaks (and minima) acquire a period which rapidly converges to twice the coherence length, $L_{\mathrm{coh}}=\frac{\pi}{|\Delta k|}=6.28 \mathrm{~cm}$. This overall periodicity is very similar to that displayed in figure 2 based on the plane-wave approximation (eqn. (1)), and might be expected given the common $\exp (\mathrm{i} \cdot \Delta k z)$ term in both eqns. (1) and (13). However, for the case of a focused beam, the amplitude of each subsequent peak clearly diminishes with $2 L$, and approaches zero as $L \rightarrow \infty$ as predicted by eqn. (13) [13]. Finally, one observes that for a constant $L, \frac{\Lambda \prime}{z_{R}}$ generally increases with $z_{R}$, a result which happens to concur with predictions based on the modified plane-wave approximation, (i.e. figure 3(a)).

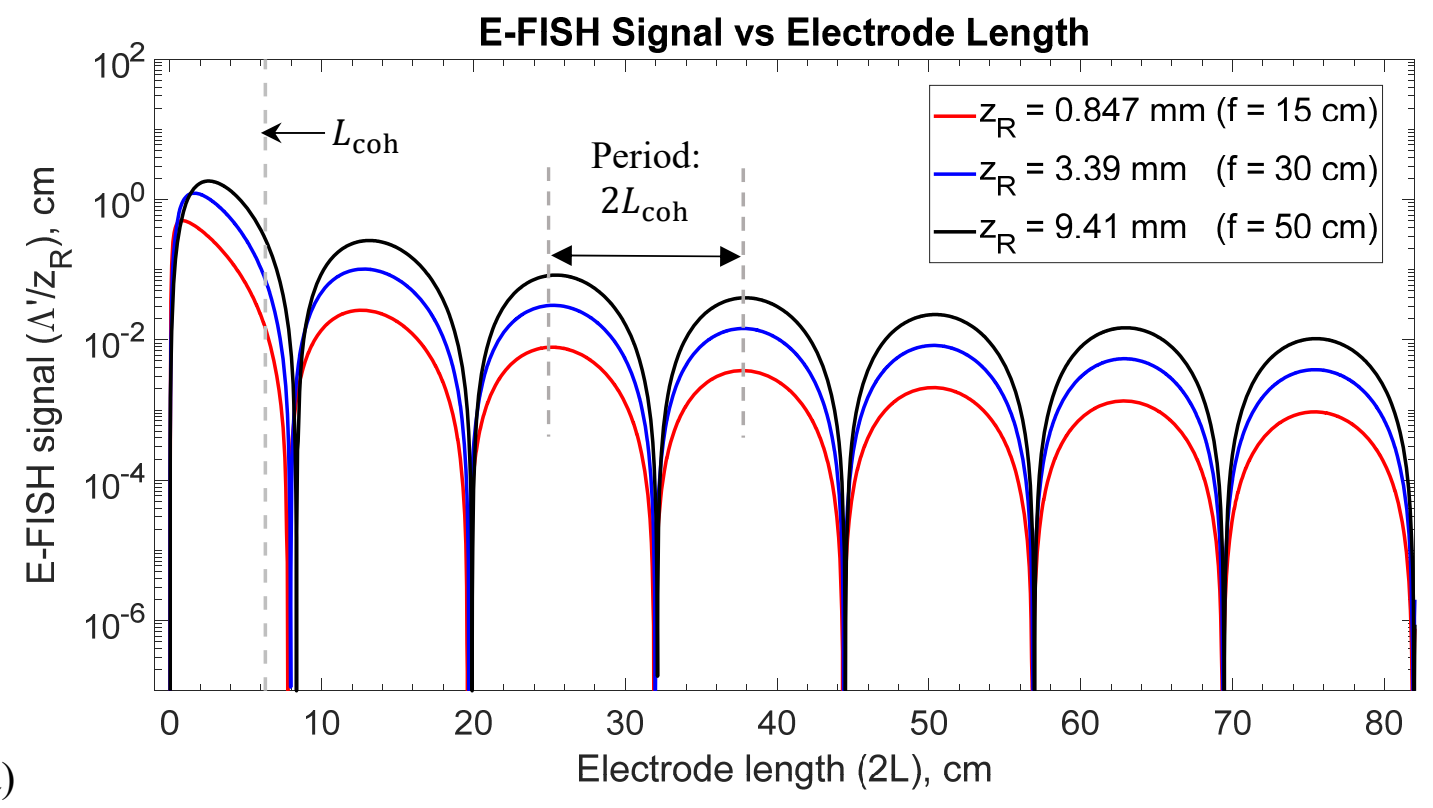




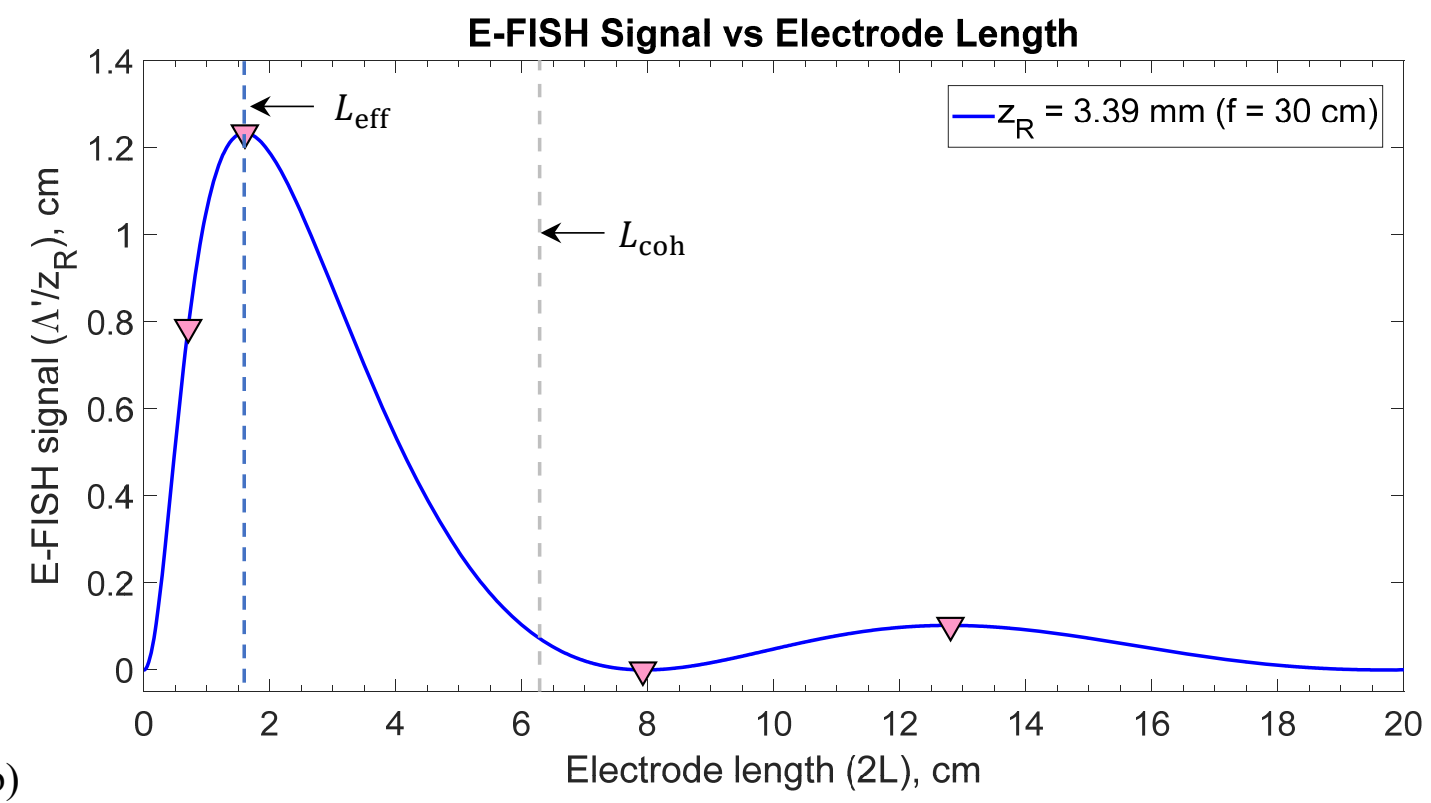

Figure 4. (a) Effect of electrode length $2 L$ on $\frac{\Lambda^{\prime}}{z_{R}}$ (viz. E-FISH signal) for three different values of $z_{R}$ (note the logarithmic scale on the vertical axis). Corresponding values of the parameter $u$ are $u=-0.0423,-0.169$ and -0.47 . (b) Effect of electrode length $2 L$ on $\frac{\Lambda^{\prime}}{z_{R}}$ for a constant $z_{R}=$ $3.39 \mathrm{~mm}$ (reproduced from (a) on a linear scale). The inverted triangles indicate the electrode lengths studied in section 2.4 and figure 6 . In both plots, $\Delta k=-0.5 \mathrm{~cm}^{-1}$, and the vertical blue and gray dashed lines represent $L_{\text {eff }}$ and $L_{\text {coh }}$ respectively.

\subsection{Effect of focused beams on the coherence length}

The results in figure 4(a) reveal an interesting trend in relation to the coherence length, $L_{\text {coh }}=$ $\frac{\pi}{|\Delta k|}$. As specified above, the location of the first signal peak, which we define as $L_{\text {eff }}$, occurs at an electrode length which is significantly smaller than $L_{\text {coh }}$. We find empirical evidence that this occurs when the parameter $|u|$, where $u=\Delta k \cdot z_{R}$ is small, for e.g. less than unity (as in figure 4) which is typical of E-FISH experiments in a gas. Note that in figure 4, the corresponding values of the parameter $u$ for $z_{R}=0.847 \mathrm{~mm}, 3.39 \mathrm{~mm}$ and $9.41 \mathrm{~mm}$ are $u=-0.0423,-0.169$ and 0.47 respectively. However, at larger values of $|u|$ more typical of solid media such as glass where the phase-mismatch $\Delta k$ is much greater than in air, $L_{\text {eff }} \rightarrow L_{\text {coh }}$. A similar result is expected to apply in a plasma as the working pressure is increased, since $\Delta k$ is directly proportional to the gas number density, through the index of refraction.

As evidence of this behavior, we plot in figure 5 the E-FISH signal for three distinct values of $u$, namely $u=-0.169,1.69$ and 16.9 . Since $u=\Delta k \cdot z_{R}$, an increase in $|u|$ may be viewed in terms of an increase in either $z_{R}$ (figure 5(a)) or $|\Delta k|$ (figure 5(b)) with the other variable held constant. In both plots of figure 5 , it is clearly seen that $L_{\mathrm{eff}} \rightarrow L_{\mathrm{coh}}$ as $|u|$ is increased, which suggests that 
the result for the plane-wave approximation is recovered when $|u|$ (i.e. $z_{R}$ or $|\Delta k|$ ) becomes large. In this context, the effect of an increase in $z_{R}$ is faithfully predicted by eqn. (13) and has been explained earlier in section 2.2. On the other hand, viewing this statement in terms of an increase in $|\Delta k|$ is slightly less apparent. However, one notes that as $|\Delta k|$ is increased, the coherence length becomes smaller and begins to converge to $L_{\text {eff. }}$. In essence, the effects of dispersion become important over a length scale that is comparable to or smaller than the focal region (viz. $z_{R}$ ). Related ideas and use of the dimensionless parameter $|u|$ have also been reported in the literature for characterizing third-order nonlinear processes [16, 17] and figure 7.4 of [18].

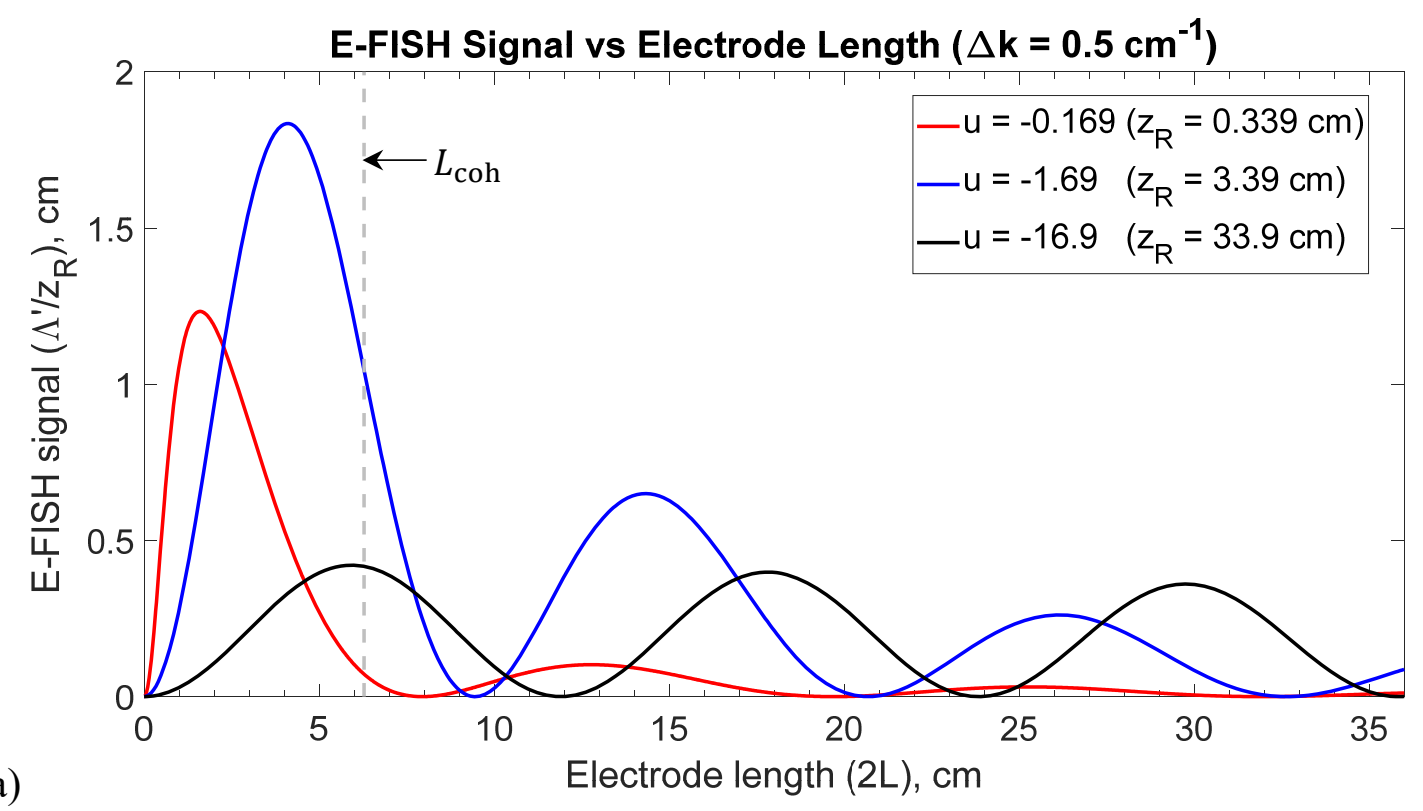

(a)

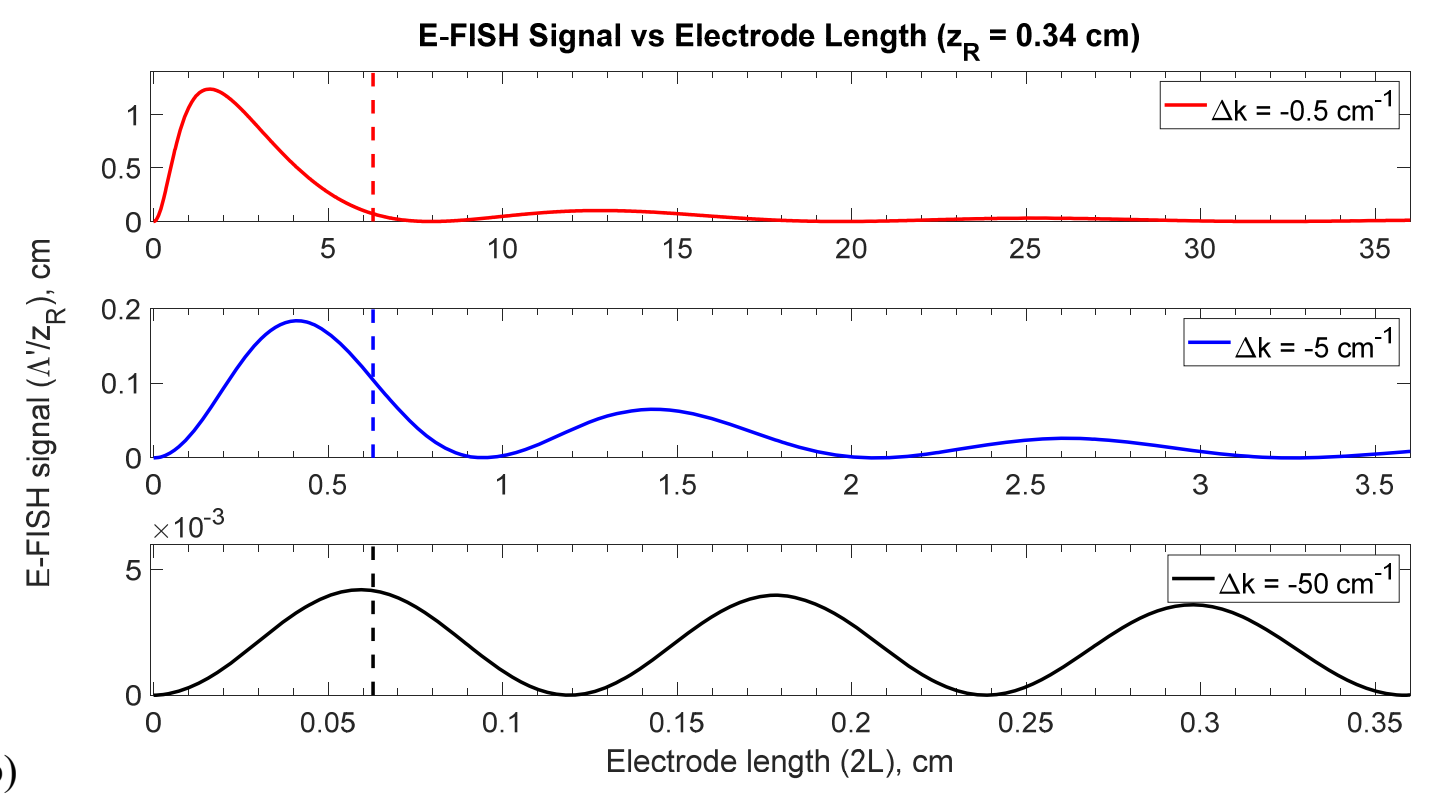


Figure 5. Effect of the parameter $u$ on the E-FISH signal, viewed in terms of a variation in (a) the Rayleigh range, $z_{R}$; and (b) wave vector mismatch, $\Delta k$. Vertical dashed lines correspond to the location of the respective coherence lengths, $L_{\text {coh }}$.

\subsection{Spatial evolution of the E-FISH signal}

To gain further insight into the above results, we trace the spatial evolution of the E-FISH signal along the z-axis for a constant $z_{R}$ analogous to eqn. (5) and figure 3(b). This is facilitated by plotting the following equation,

$$
P^{(2 \omega)}(z) \propto \frac{\Lambda^{\prime}}{z_{R}}(z) ; \quad \text { where } \Lambda^{\prime}(z)=\left|\int_{-L}^{z} \frac{\exp (\mathrm{i} \cdot \Delta k z)}{\left[1+\mathrm{i} \cdot\left(\frac{z}{z_{R}}\right)\right]} \mathrm{d} z\right|^{2} \text { for }-L \leq z \leq L .
$$

Figure 6 shows the spatial evolution of the E-FISH signal for 4 different electrode lengths $(2 L)$, and $z_{R}=3.39 \mathrm{~mm}$. These electrode lengths are chosen such that they mainly coincide with respective maxima and minima of $\frac{\Lambda^{\prime}}{z_{R}}$ as indicated by the inverted triangles in figure $4(\mathrm{~b})$. They have also been found to be representative of the results for other $z_{R}$. It is observed from figure 6(a) that for the 2 selected electrode lengths where $2 L \leq L_{\text {eff }}$ of figure 4(b), the signal buildup along the $z$ direction is essentially confined to the beam focal region. However, for the other 2 cases where $2 L>L_{\text {eff }}$, an interesting profile is observed, in contrast to that of figure $3(\mathrm{~b})$ for the same electrode length. The signal builds up sharply as one approaches the beam focus from $z<$ 0 , but displays an equally pronounced decline for $z>0$. The final value of the signal is therefore substantially weaker than at its peak, and is also affected by changes in the far field $\left(z>\left|z_{R}\right|\right)$.

We attribute this steep rise and fall near the beam focus to constructive and destructive interference, which occurs due to the cumulative contribution of the Gouy phase shift $\phi_{\mathrm{g}}$ to the phase of the E-FISH field. This Gouy phase shift acts as a supplementary contribution to the total phase mismatch, in addition to $\Delta k z$. To have a better insight about this effect, we remember that the phase mismatch in nonlinear optics is the difference between the phases of the fundamental and harmonic beams. Mathematically, the total (or net) phase mismatch of the E-FISH field, $\Delta \phi_{\text {tot }}(z)$, may be written as:

$$
\begin{gathered}
\Delta \phi_{\text {tot }}(z)=\left[2 k^{(\omega)}-k^{(2 \omega)}\right] \mathrm{z}-\left[2 \phi_{\mathrm{g}}^{(\omega)}(z)-\phi_{\mathrm{g}}^{(2 \omega)}(z)\right] \\
=\Delta k . z-\phi_{\mathrm{g}}(z)
\end{gathered}
$$

The r.h.s of eqn. (15) therefore consists of two difference contributions - one due the regular wave vector mismatch, $\Delta k$, and the other due to the difference in the Gouy phase shifts. The second term involves subtracting the Gouy phase shift of the E-FISH beam from that of the fundamental beam, the latter being multiplied by 2 to take into account the fact that we are considering SHG. Since the electric fields of the E-FISH signal and the probe beam have the same Rayleigh range as noted earlier in section 2.2, both the fundamental and second harmonic waves acquire the same 
Gouy phase shift (i.e. $\phi_{\mathrm{g}}^{(\omega)}=\phi_{\mathrm{g}}^{(2 \omega)}$ ). Thus the supplementary phase mismatch corresponds to one Gouy phase shift along the interaction length $2 L$.

The spatial evolution of the signal is therefore influenced by both the spatial evolution of the laser intensity (which is well accounted for in the modified plane wave approximation), as well as that of the total phase mismatch, which includes the supplementary contribution from the Gouy phase shift. We remark that the Gouy phase shift increases by $\pi\left(-\frac{\pi}{2}\right.$ to $\left.\frac{\pi}{2}\right)$ from $z=-\infty$ to $z=+\infty$, switching sign as one crosses the beam focus. This sign change in $\phi_{\mathrm{g}}$ across the beam focus is responsible for the signal evolution shown in figure 6(b). For small $L$ (figure 6(a)), the signal does not build up over a sufficient distance for destructive interference to occur, and the effect of the phase mismatch on the signal is weak compared to the stronger intensity within the focal region. The shape of the evolution curve thus appears similar to that observed in the modified plane wave approximation. At larger $L$ (figure 6(b)), the phase mismatch contribution due to $\phi_{\mathrm{g}}(z)$ negates part of the signal contribution from the higher intensity. The eventual path-integrated signal attains a value between either a null or a local maximum, and explains the periodic dependence of $\Lambda^{\prime}$ on the electrode length. At these longer electrode lengths, this final signal appears to be influenced by both the interaction within the focal region, and the far field. As a final note, we emphasize that the main difference in the results obtained in this section versus the modified plane-wave approximation, is due to the Gouy phase shift, which profoundly alters the interaction between the fundamental and second harmonic waves. The effects of this Gouy phase shift, which become more pronounced with stronger focusing, are neither applicable to plane waves, nor captured in the modified plane-wave approximation.

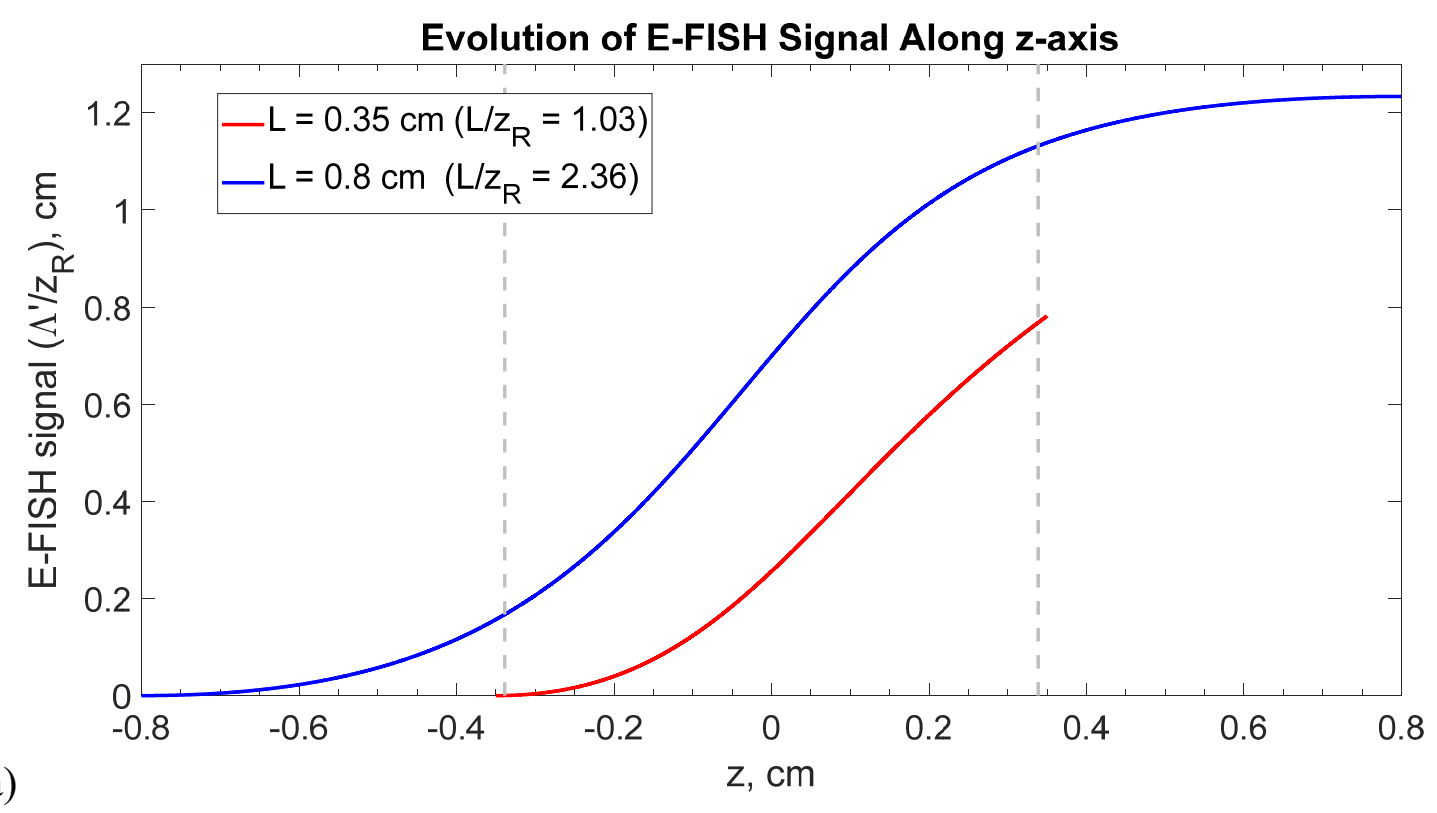




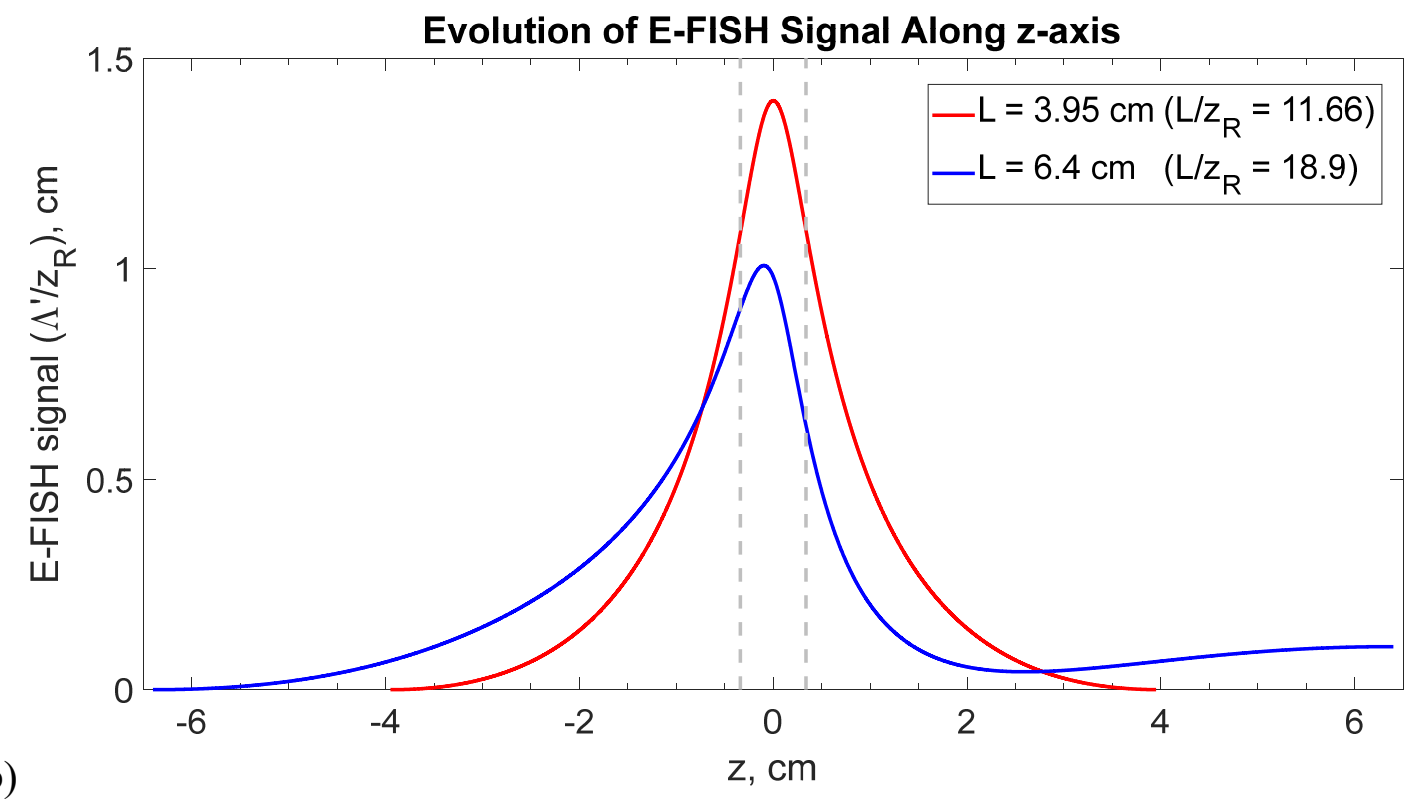

Figure 6. Spatial evolution of $\frac{\Lambda^{\prime}}{z_{R}}$ along the $z$-axis for a) two smaller values of $L \leq L_{e f f}$, b) first maximum and minimum for $L>L_{\text {eff }}$. In both plots, $z_{R}=3.39 \mathrm{~mm}$ and $\Delta k=-0.5 \mathrm{~cm}^{-1}$. Gray vertical dashed lines correspond to $z= \pm z_{R}$.

\subsection{Effect of a spatially varying externally applied electric field}

In the previous sections, we have considered the effect of a constant external field, $E_{\text {ext }}$. Here, we briefly examine the influence of a spatially varying external field, $E_{\text {ext }}(z) \propto \frac{1}{1+4 \cdot\left(\frac{z}{\delta}\right)^{2}}$, which is more representative of reality. This Lorentzian function mimics closely the field distribution from two cylindrical electrodes as studied in [19], where $\delta$ effectively determines the length (or full width at half-maximum (FWHM), see inset of figure 7) of the external electric field profile. As mentioned in section 2.2, the effect of a $z$-varying external field may be studied by incorporating a function $E_{\text {ext }}(z)$ into eqn. (7), leading to a modified version of eqn. (13) (note the limits of the modified integral), given by:

$$
P^{(2 \omega)} \propto\left(\frac{\Lambda^{\prime \prime}}{z_{R}}\right) ; \text { where } \Lambda^{\prime \prime}=\left|\int_{-\infty}^{\infty} \frac{\exp (\mathrm{i} \cdot \Delta k z)}{\left[1+\mathrm{i} \cdot\left(\frac{Z}{z_{R}}\right)\right]} \cdot \frac{1}{\left[1+4 \cdot\left(\frac{z-z_{\mathrm{e}}}{\delta}\right)^{2}\right]} \mathrm{d} z\right|^{2}
$$

where $\delta$ is a parameter which determines the length (or FWHM) of the external electric field profile, centered at $z=z_{\mathrm{e}}$, and all other terms are as previously defined. 


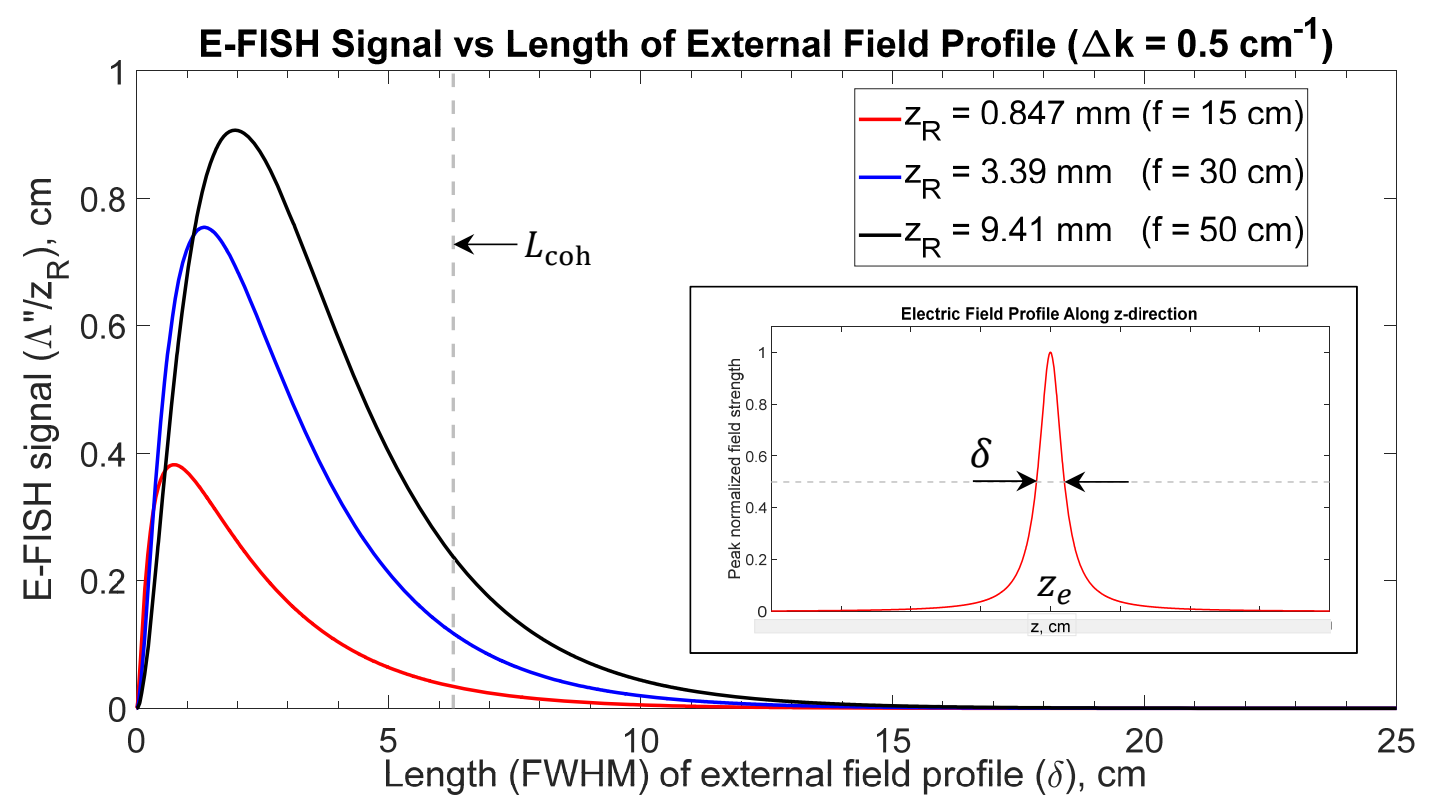

Figure 7. Effect of the length of the external field profile, $\delta$ on the E-FISH signal $\left(\frac{\Lambda^{\prime \prime}}{z_{R}}\right)$, with $z_{\mathrm{e}}=0$ for three different values of $z_{R}$ and $\Delta k=-0.5 \mathrm{~cm}^{-1}$. Inset: shape of the externally applied electric field, indicating the FWHM (width of the field profile where $E_{\text {ext }}$ drops to half its peak value) defined by $\delta$.

Again, we remark as before, that the effect of the spatially varying external field profile on the EFISH signal is fully captured by the parameter $\frac{\Lambda^{\prime \prime}}{z_{R}}$ and shown in figure 7 . In a similar manner to figure 4 , a dependence on both $z_{R}$ and the spatial extent of the external field, $\delta$ is clearly identified, with the main difference being that the signal periodicity is no longer detected. The reason for this disappearance is attributed to the fact that the effects in the far field become weaker due to the decreasing field strength. In particular, one notes that as $\delta \rightarrow \infty, \Lambda^{\prime \prime} \rightarrow 0$. Another important inference from figure 7 is that the shape of the electric field profile also has an impact on the resulting signal. Comparing figures 4 and 7 , one finds that the peak signals produced with a constant field are consistently larger than those with a spatially varying field. While this is perhaps unsurprising given the lower spatially averaged field in the case of the latter, it is also interesting to note that the effective coherence lengths $L_{\text {eff }}$, for the spatially varying field (figure 7) are slightly shorter than the ones for the uniform field (figure 4 ). However, in both cases, $L_{\mathrm{eff}}$ is always smaller than $L_{\text {coh }}$. Further discussions on how the electric field shape affects the signal may be found in the later part of the following section, as well as in section 3.2.

\subsection{Spatial resolution of an E-FISH measurement}

The conclusions drawn from figures 4 and 6 naturally provoke the question as to what the true spatial resolution of an E-FISH measurement is. While we have shown that the signal indeed depends on the entire spatial profile of the external electric field, it is equally important to note 
that the signal is also most sensitive to changes in the field strength near the beam focus (see for instance figure 6). More specifically, we refer again to the canonical electrode geometry in figure 1(a). For a fixed electrode length $2 L$, it is found that the E-FISH signal is much more sensitive to a local increase in $E_{\text {ext }}$ imposed near the beam focus, as compared with a local increase imposed in the far field. In this sense, one may cautiously concede that the spatial origin of the signal is still associated with the confocal region (i.e. $2 z_{R}$ ). This idea also finds support in previous studies, which involve translating the probe beam along the $z$ direction with respect to the external field. These studies have found that the E-FISH signal profile as a function of $z$ exhibits a length scale given by the convolution between the spatial profile of the external field and the confocal parameter of the probe beam $[1 \& 6]$.

At the same time, one should be cognizant of certain exceptions. Suppose we consider the same geometry in figure 1(a), but with a spatial void in the electrodes of length $2 a$, centered at $z=0$, as shown in figure $8(\mathrm{a})$. Such a field distribution is relevant to spatially non-uniform or filamentary discharges such as that in [20 \& 21], where regions of strong and weak electric field are distributed throughout the plasma. In ref. [21] for instance, a filament with high electron density $\left(10^{19} \mathrm{~cm}^{-3}\right)$ and thus low electric field, is surrounded by a streamer zone with a high electric field. Likewise, such spatially varying fields are also associated with the passage of fast ionization waves in nanosecond discharges [3], especially in the region behind the ionization front.

(a)

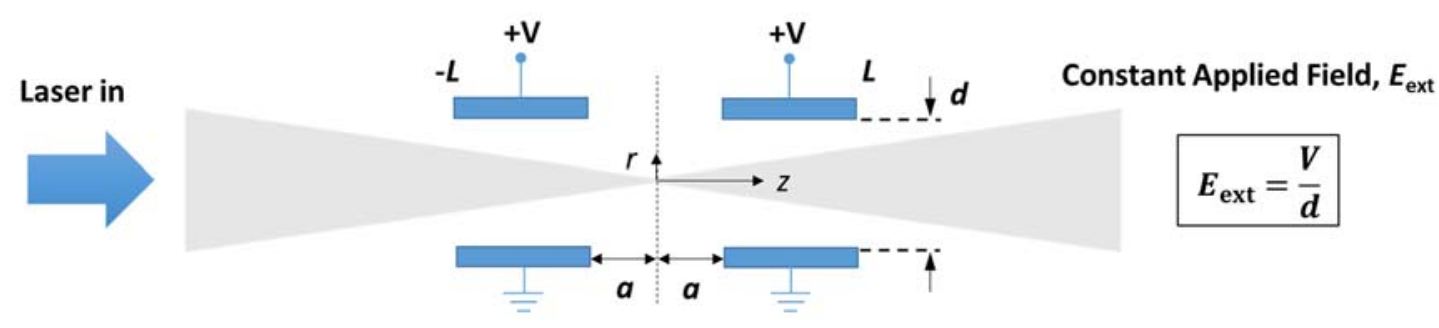

(b)

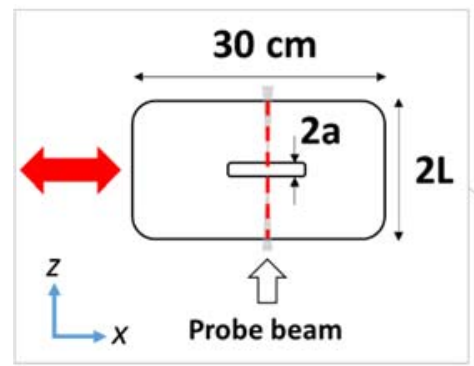

Probe beam

$$
\begin{array}{l|c}
\text { Electrode length, 2L } & 14.5,22 \mathrm{~cm} \\
\hline \text { Void length, 2a } & 1 \mathrm{~cm}
\end{array}
$$

$2 \mathrm{kV}$

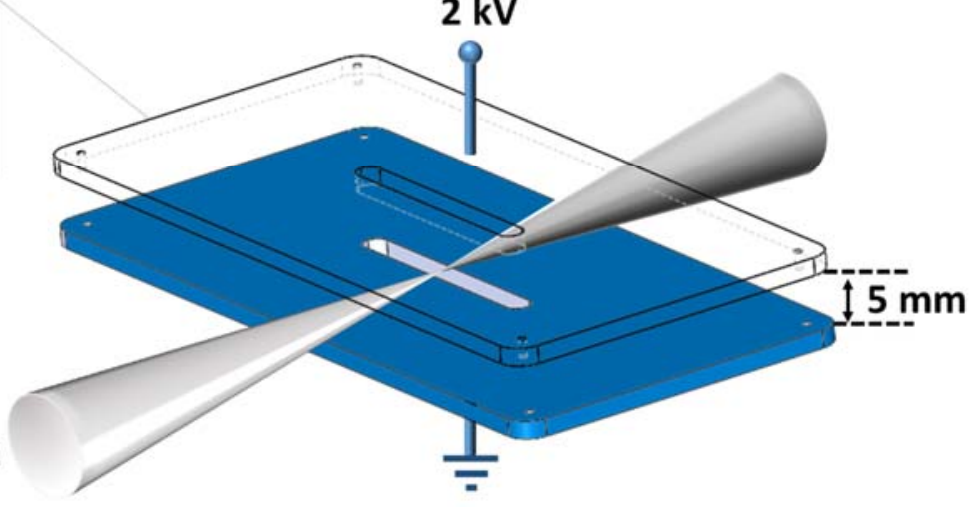


Figure 8. (a) Schematic of electrode geometry for assessing the spatial resolution of the E-FISH signal. (b) Corresponding schematic of the electrode geometry used in the experiments. Translating the electrodes along the direction of the red arrow (x-axis) in the figure inset facilitates a comparison with the baseline signal (i.e when there is no void).

To assess such a configuration, the integral on the r.h.s of eqn. (13) is split into 2 parts, as shown below in eqn. (17), and evaluated for $0 \leq a \leq L$, for two different values of $L$ and a constant $z_{R}$ as shown in figure 9 .

$$
P^{(2 \omega)} \propto\left(\frac{\Lambda^{\prime \prime \prime}}{z_{R}}\right) ; \text { where } \Lambda^{\prime \prime \prime}=\left|\int_{-L}^{-a} \frac{\exp (\mathrm{i} \cdot \Delta k z)}{\left[1+\mathrm{i} \cdot\left(\frac{Z}{z_{R}}\right)\right]} \mathrm{d} z+\int_{a}^{L} \frac{\exp (\mathrm{i} \cdot \Delta k z)}{\left[1+\mathrm{i} \cdot\left(\frac{z}{z_{R}}\right)\right]} \mathrm{d} z\right|^{2}
$$

The pair of selected $L$ values correspond approximately to electrode lengths which produce a signal minimum $(2 L=22 \mathrm{~cm})$ and local maximum $(2 L=14.5 \mathrm{~cm})$ in the experiments (see figure 10$)$. An interesting and somewhat counter-intuitive result emerges from these theoretical calculations. It turns out that for certain values of $a$, an increase in the E-FISH signal of up to an order of magnitude is realized. This is in fact in contradiction to our earlier statement regarding the spatial origin of the signal. We attribute this signal increase to the effect of the Gouy phase shift as in the signal profiles shown in figure 6(b). The addition of a void (or null in the electric field) acts to eliminate the constructive and destructive interference such that the eventual signal is in fact higher than if this null were to be absent.

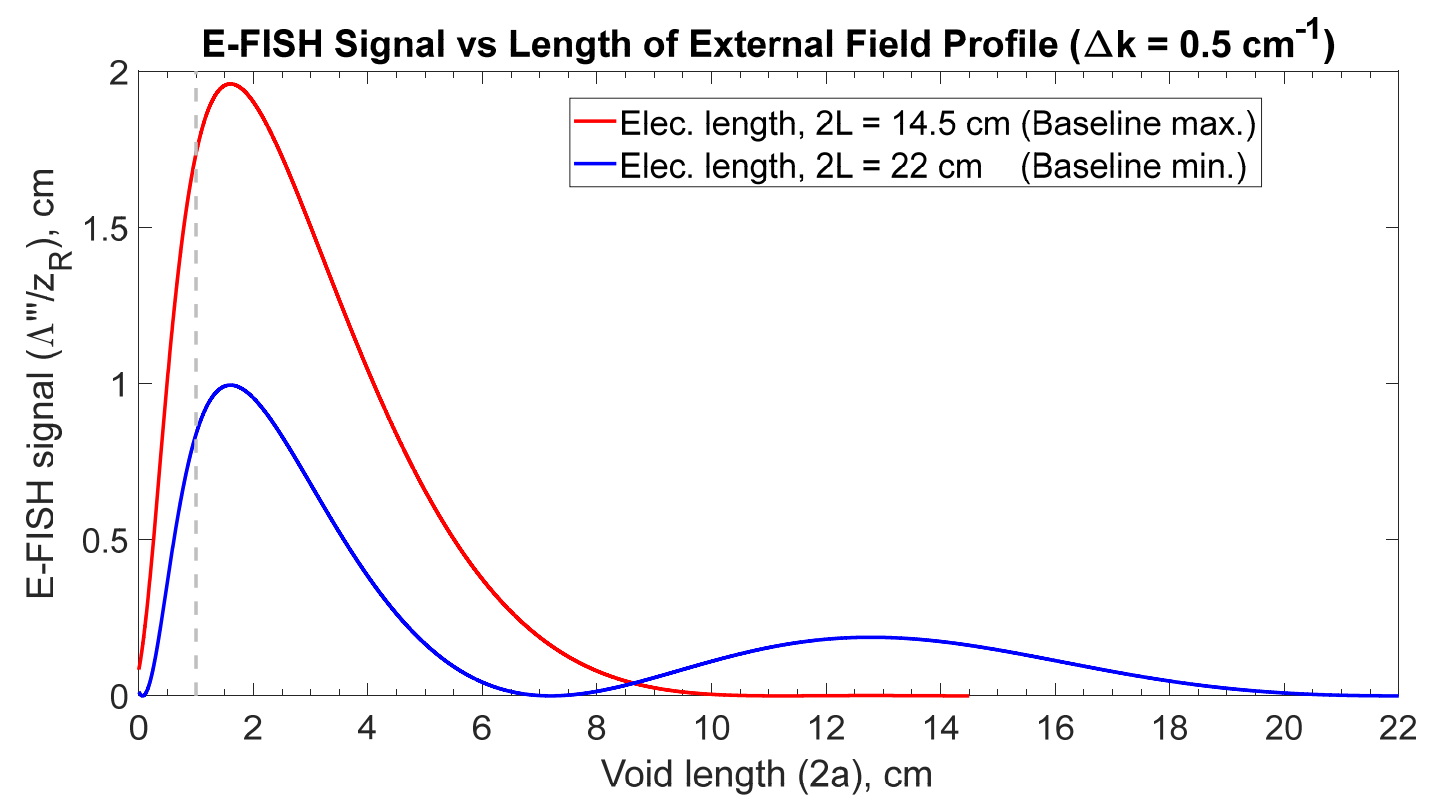

Figure 9. Effect of void length, $2 a$ on the E-FISH signal for two different electrode lengths $2 L=$ $14.5 \mathrm{~cm}$ and $22 \mathrm{~cm}$, for $z_{R}=3.39 \mathrm{~mm}$ and $\Delta k=-0.5 \mathrm{~cm}^{-1}$. Gray vertical dashed line indicates a void length, $2 a$, of $1 \mathrm{~cm}$ used in the corresponding experiments. Note that the signals for $2 a=$ 0 correspond to the baseline values where there is no void. 


\section{Experimental results and discussion}

\subsection{Effect of electrode length on E-FISH signal}

The observed dependence of the E-FISH signal on the entire spatial profile of $E_{\text {ext }}$ in sections 2.2 and 2.6 poses a genuine challenge for obtaining accurate, absolute electric field data. As earlier discussed, most of the existing experiments rely on signal calibration using a known, electrostatic field, then apply this calibration to the signals acquired in a plasma. A dependence of the signal on the external field profile would demand matching field profiles during calibration and in the plasma, to ensure a good measurement. Since the spatial extent of $E_{\text {ext }}$ in a plasma is often unknown, this would further complicate the calibration procedure. It is therefore imperative to provide an experimental verification of these theoretical predictions.

To this end, we perform an E-FISH experiment with a pair of stainless steel, triangular parallelplate electrodes as shown in figure 1(b). The interelectrode gap distance is $5 \mathrm{~mm}$, and a constant external electric field is sustained across the gap via a $2 \mathrm{kV} \mathrm{DC}$ voltage. Translating the electrodes along the $x$-axis, and measuring the vertically polarized (parallel to $E_{\text {ext }}$ ) E-FISH signal at each $x$ position, provides a convenient way of assessing the effect of electrode length. A $3 \mathrm{~ns}, 4 \mathrm{~mJ}, 1064$ $\mathrm{nm}$ beam, with a pulse repetition rate of $10 \mathrm{~Hz}$, is extracted from a Nd:YAG laser using an Pockelscell-based optical pulse slicer [10]. This probe beam is passed along the mid-height of the interelectrode gap (i.e. $\sim 2.5 \mathrm{~mm}$ ), and focused at the center of the electrodes (i.e. at the $x$-axis) using a plano-convex spherical lens. The resulting E-FISH signal is collimated with a second spherical lens, and focused with a third lens onto a photomultiplier (PMT). A polarizing beamsplitter placed before this third spherical lens ensures that only the vertically polarized component of the signal is recorded. A photodiode with a $1 \mathrm{~ns}$ response time is used for monitoring the laser intensity. Other details of the experimental setup can be found in [10] and are omitted for the sake of brevity.

Figure 10 shows the E-FISH signal as a function of electrode length acquired using three different lenses with focal lengths of $15 \mathrm{~cm}, 30 \mathrm{~cm}$ and $50 \mathrm{~cm}$. While not directly measured, the respective estimated Rayleigh ranges are $z_{R}=0.847 \mathrm{~mm}, 3.39 \mathrm{~mm}$ and $9.41 \mathrm{~mm}$, based on a Gaussian beam approximation for our initial (collimated) beam waist of $3 \mathrm{~mm}$. These values match exactly those studied in section 2. The results of these experiments are in good agreement with the theoretical predictions of figure 4(a); the E-FISH signal displays an oscillatory dependence on the electrode length, decreasing gradually with increasing electrode length as predicted by eqn. (13). Subsequent minima also display a period of roughly $12 \mathrm{~cm}$, corresponding to twice the coherence length, $L_{\text {coh }} \approx 6 \mathrm{~cm}$, just as in figure 4(a). In particular, the slight difference in the location of the local maxima and minima for $z_{R}=0.847 \mathrm{~mm}$ versus $z_{R}=3.39 \mathrm{~mm}$ and $9.41 \mathrm{~mm}$ seen in figure 4(a) is also captured in the experiments. This shift is likely to be due to differences in the effective coherence length, $L_{\text {eff }}$ as discussed in section 2.3. Finally, an imperfect correspondence of the

precise locations of the minima and maxima between theory and experiment is also noticed. This is anticipated to be a combination of field edge effects, which can alter the uniform shape and 
length of the external field profile, as well as the measurement uncertainty in the actual electrode length (estimated to be $2 \mathrm{~mm}$ ) probed by the laser beam.

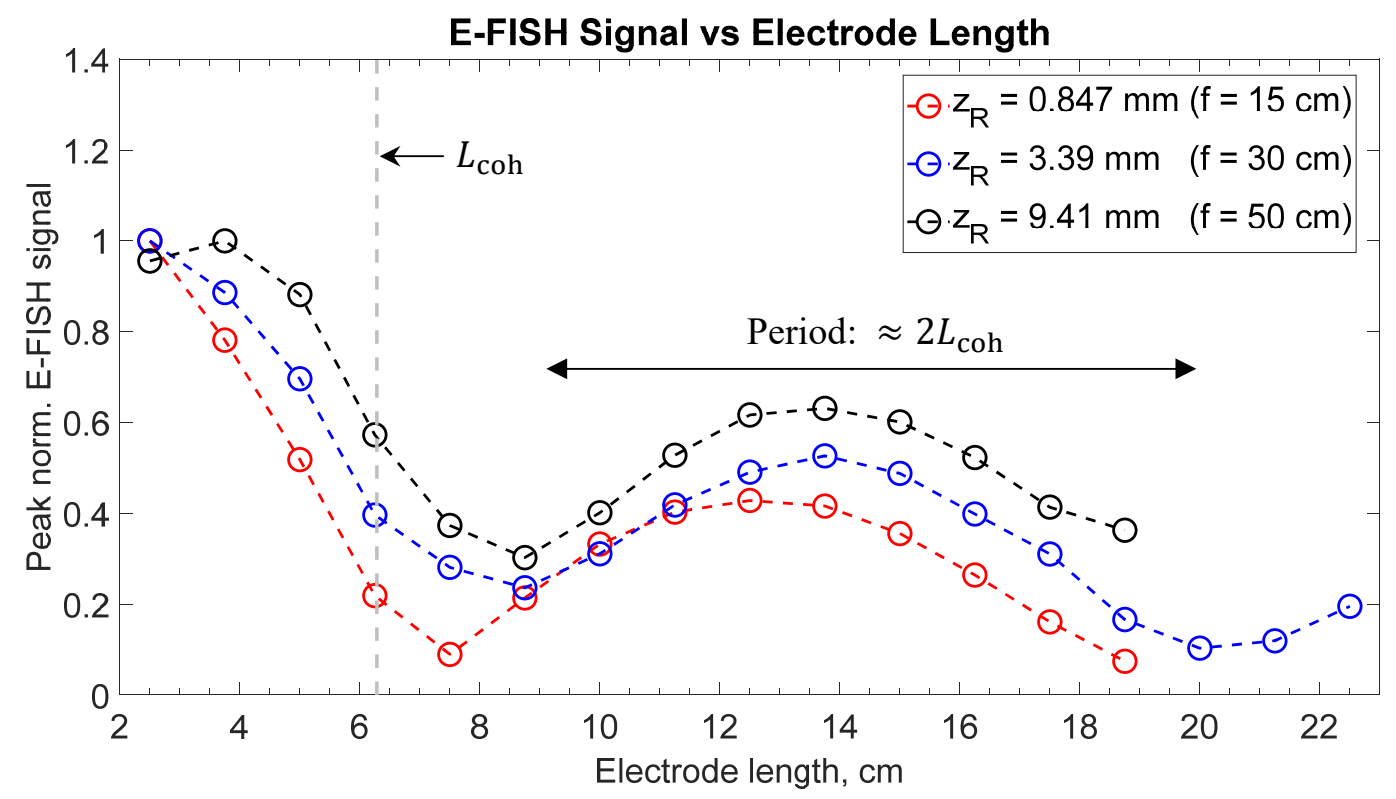

Figure 10. Effect of electrode length on the peak normalized E-FISH signal obtained using 3 lenses of different focal lengths (viz. $z_{R}$ ). The uncertainty in the data with respect to the horizontal axis is about $2 \mathrm{~mm}$.

In order to rule out the possibility that the observed signal periodicity originates from changes in the external electric field (for e.g. edge effects) instead of the variation in electrode length, a numerical simulation of the electrostatic field between the two triangular electrodes is performed by solving the Laplace equation. Figure 11 shows that the electric field strength is indeed uniform over almost the entire electrode (except for a few $\mathrm{mm}$ at the edges), and certainly within the confocal parameter of the 3 different lenses.
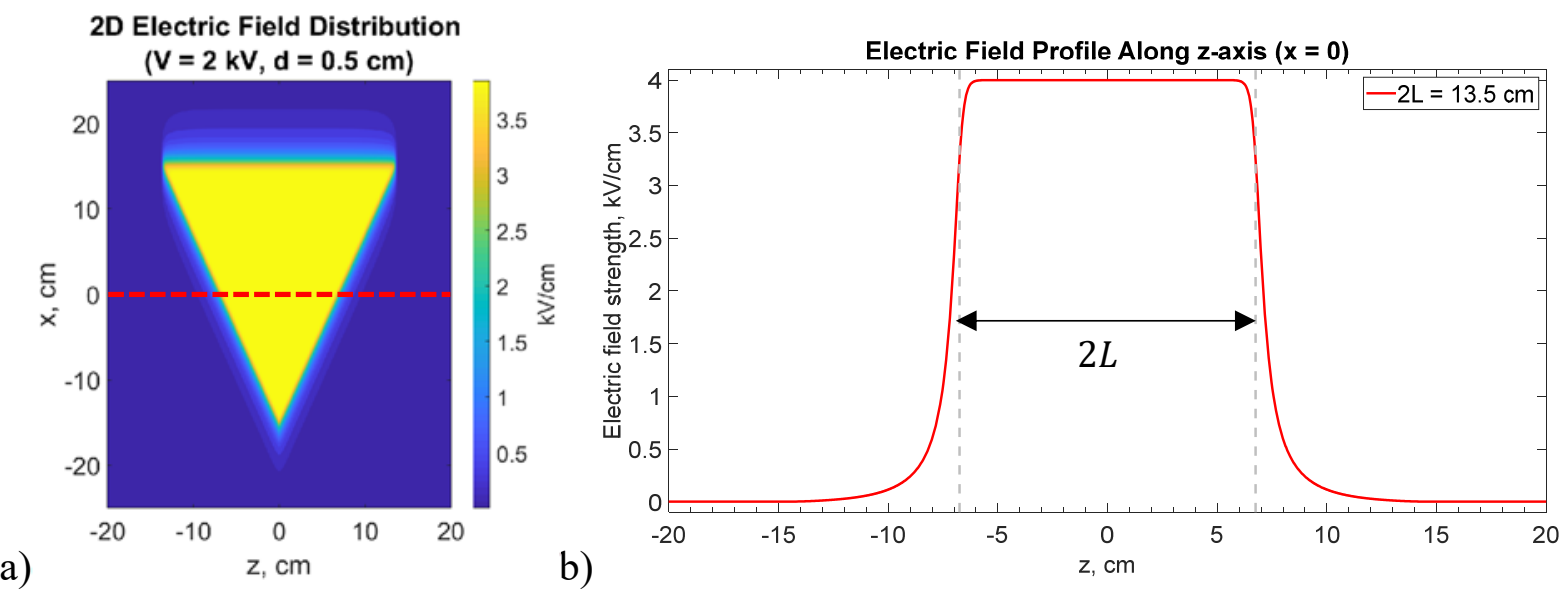

Figure 11. a) Simulation results of the 2-D electric field strength distribution within the x-z plane, at mid-height, between two triangular electrodes, with dimensions matching those of the 
experiment. b) Sectional slice of the electric field strength (indicated by the red horizontal dashed line in (a)) at $x=0$. The corresponding electrode length at this location is $13.5 \mathrm{~cm}$.

\subsection{Effect of a spatial void in the external electric field profile}

An experimental verification of the theoretical results obtained with the spatial void (figure 9) is performed with the help of the rectangular, parallel-plate electrode geometry shown in figure 8(b). The electrodes are translated along the x-axis (red arrow in the inset of figure 8(b)) in order to record the baseline values (i.e. without a void). While the full range of void lengths (in figure 9) are not tested, the primary objective of these experiments is to confirm that one can indeed induce an increase (rather than drop) in the E-FISH signal with a field null near the focal region. The choice of void size is dictated by two opposing considerations: too large a void may reduce the possible signal increase, while a void which is too small may suffer from field edge effects. The value of $1 \mathrm{~cm}$ is chosen as a balance between these two considerations. To minimize edge effects, all the electrode edges are rounded rather than sharp. Furthermore, even in the unavoidable presence of edge effects, the electric field along the z-axis at $r=0$ within the void region is anticipated to be less than the applied voltage of $2 \mathrm{kV}$. (This point has been verified using electrostatic simulations.)

In qualitative agreement with theory, the PMT time traces in figure 12 show that a $1 \mathrm{~cm}$ void located at the beam focus produces a signal increase for two different electrode lengths. If the EFISH signal originates primarily from the focal region, a drop in signal - rather than any increase - would be expected. That a drop in signal is indeed observed, provides further evidence that interactions in the far field play a significant role in affecting the E-FISH signal. It also supports the notion that the shape of the electric field has an influence on the signal.

On a separate note, the presence of edge effects could be a reason why there is imperfect quantitative agreement between theory and experiment in terms of the magnitude of the signal increase. This issue is currently a subject of ongoing research. Finally, the difference in the baseline signals (i.e. without a void) for the two electrode lengths also lends further confidence to the results obtained with the triangular electrodes in figure 10. 


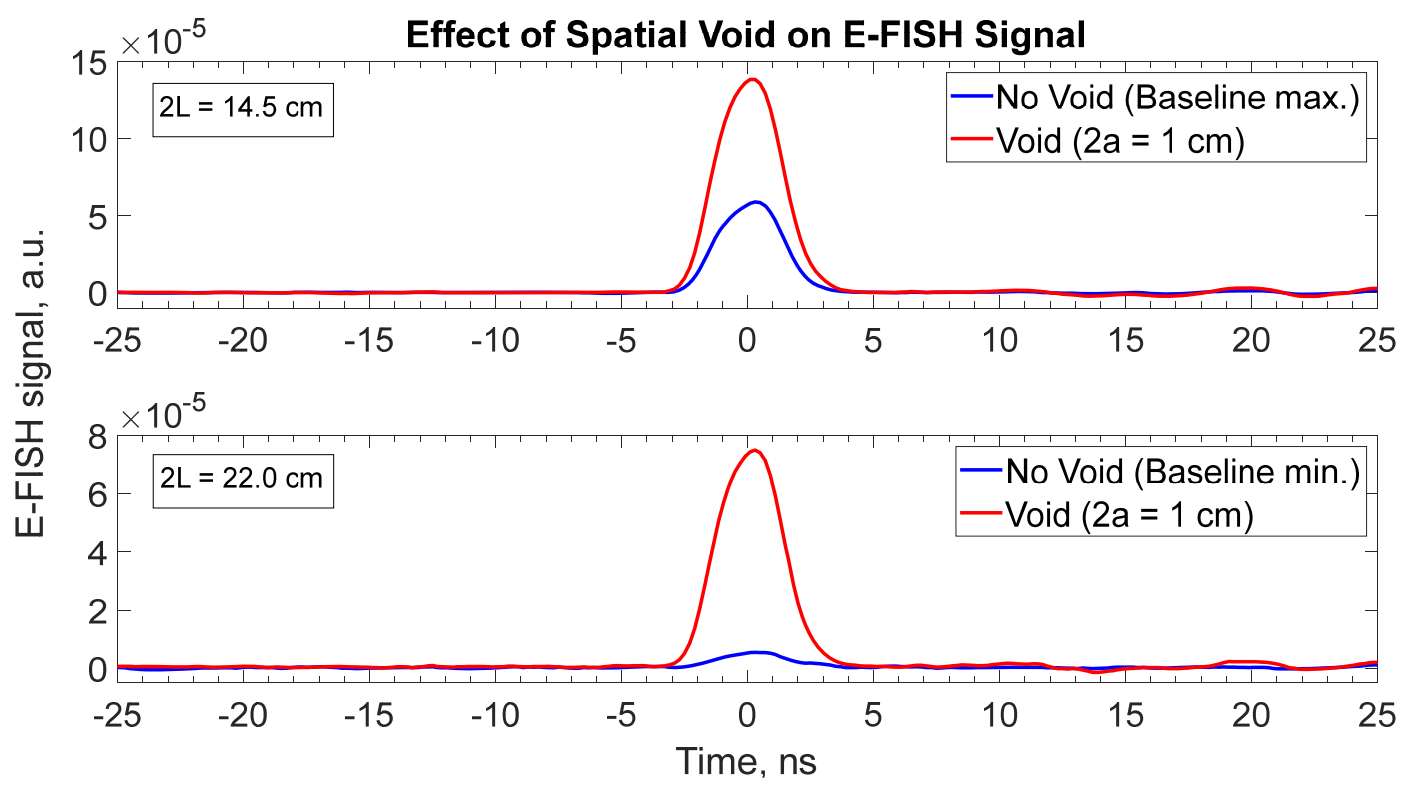

Figure 12. 300-shot averaged PMT time traces (normalized by the laser intensity, viz. timeintegrated photodiode signals) for two different electrode lengths $(2 L=14.5 \mathrm{~cm}$ and $22 \mathrm{~cm})$, showing an increase in signal with a $1 \mathrm{~cm}$ void.

\section{Discussion}

\subsection{Importance of Gouy phase shift in E-FISH diagnosis}

Taken together, the results from the previous two sections provide a sound indication that the E-FISH signal obtained using a focused beam is not only dependent on the magnitude of the external field as previously understood, but also on the spatial profile (length and shape) of this external field. The physical reason for this behaviour is that the Gouy phase shift exhibited by focussed beams modifies the phase matching of the E-FISH process. Similar effects have been observed in SHG and THG microscopies [22-29]. SHG microscopy obeys the same equation as EFISH, except that the product of the third-order hyperpolarizability and the external electric field $\alpha_{i j k l}^{(3)} \cdot\left(E_{\text {ext }}\right)_{j}$ is replaced by the second-order hyperpolarizability $\beta_{i k l}^{(2)}$ in equation (3). This tensor is non-zero only for non-centrosymmetric media [13], which is a very stringent condition and explains why E-FISH is used in isotropic media. SHG microscopy has been mostly applied to biological tissues and only a few biological components have been found to exhibit SHG signal: fibrillary collagen, myosin filaments in muscles and dense arrays of microtubules [30, 31]. As these non-centrosymmetric structures have small dimensions, the SHG signal evolution is close to the figure 6(a) curves and the Gouy phase shift has not as much of an effect as it does in longer media on the order of the electrode lengths shown in figure 6(b). In contrast, the Gouy phase shift has a crucial effect in THG microscopy, as already observed in gases in 1969 [14]. Indeed, while the phase matching conditions involve only one Gouy phase shift in SHG and E-FISH as explained above, it involves 2 Gouy phase shifts in THG (i.e. $3 \phi_{\mathrm{g}}^{(\omega)}(z)-\phi_{\mathrm{g}}^{(3 \omega)}(z)=2 \phi_{g}$, with reference 
to eqn. (15)) ). It means that the denominator in the integral of equation (3) is squared, and this integral is much more sensitive to smaller dimensions. One may consider that destructive interferences arise within the Rayleigh range as the Gouy phase shift between $-z_{\mathrm{R}}$ and $+z_{\mathrm{R}}$ is $\pi / 2$ (for SHG), that is $\pi$ when doubled (for THG). Accordingly, THG signals have been observed in $\mu \mathrm{m}$-scale heterogeneities in biological tissues, for instance lipid vesicles or cell interfaces [25]. EFISH diagnosis thus shares common characteristics with both SHG and THG - it exhibits the same optical behavior as SHG microscopy, specifically the interaction length dependence, but is observed only in isotropic media as with THG microscopy.

\subsection{Recommendations for E-FISH diagnosis}

To minimize any inaccuracies arising from the dependence of E-FISH signal on the spatial profile of the external field, we propose three possibilities for the consideration of future users. The first two involve experimental modifications to the E-FISH diagnostic, while the remaining suggestion retains the current working setup but attempts to implement corrections to the experimental data.

As discussed in section 2.3, one way to ensure validity of the plane-wave approximation, is to increase the Rayleigh range such that it is significantly larger than the interaction length (viz. size of the plasma). (The limit of this idea would thus be a collimated beam.) Even then, this interaction length should preferably remain below the coherence length, $L_{\text {coh }}=\frac{\pi}{|\Delta k|}$. The advantage of this approach is an increase in signal (due to a longer signal buildup path), while the evident drawback would be a loss of spatial resolution. We point out that even though this approach removes the issue of the field profile dependence on the signal, it could introduce other problem-specific inaccuracies especially if the field within the longer measurement region is spatially non-uniform or unknown. This is because the path-integrated E-FISH signal is unevenly weighted over the interaction length.

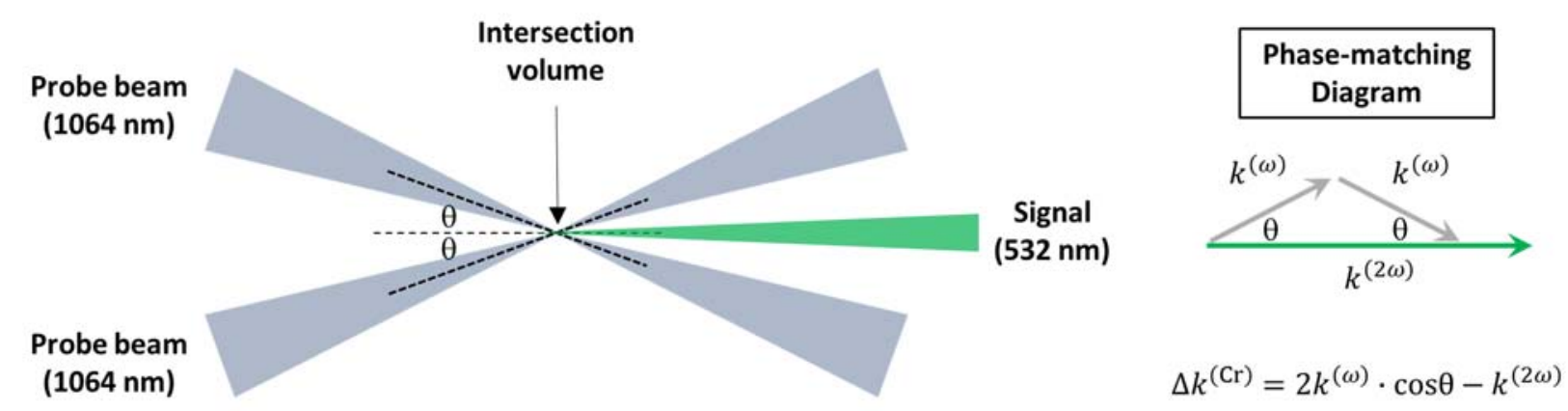

Figure 13. Schematic illustrating the concept of crossing two laser beams as a method of localizing the E-FISH measurement in space. (Note that for co-propagating beams, $\theta=0, \Rightarrow \cos \theta=1$, and $\Delta k=2 k^{(\omega)}-k^{(2 \omega)}$. Therefore $\left|\Delta k^{(\mathrm{Cr})}\right|>|\Delta k|$.) 
One way of definitively localizing the E-FISH measurement in space is to utilize two crossed laser beams instead of a single beam (see figure 13). The intersection volume between the two focused beams effectively defines the interaction length $L_{\text {int }}$, and thus the spatial resolution of the measurement. Due to phase-matching considerations, only this intersection volume generates a second harmonic signal along the angular bisector. Apart from an unambiguity in the spatial resolution, this approach provides more flexibility since the properties of each of the two beams, such as its polarization, wavelength and intensity, can be controlled separately. The main disadvantages would be a drop in signal due to a larger wave vector mismatch $\left|\Delta k^{(\mathrm{Cr})}\right| \operatorname{compared}$ with the co-propagating case, and more importantly the loss of the far field contribution to the signal. The need to align and overlap two laser beams also increases the complexity of the experimental setup. Furthermore, this approach has yet to be experimentally demonstrated, and thus warrants further examination.

The final recommendation suggests a combination of numerical modeling with E-FISH experiments to mitigate this issue. Depending on the confidence in the numerical data, these simulations could be used to provide an idea of the length and shape of the external electric field profile, $E_{\text {ext }}(z)$, which can be input into eqn. (15) in place of the Lorentzian function. This procedure can be performed for both the calibration setup and the plasma. In doing so, this offers an estimate as to how sensitive the E-FISH data is to changes in the spatial profile of the external field for a particular problem. Sensible corrections to the experimental data may then be implemented at the discretion of the user, while still retaining certain advantages of the method such as its simplicity and superior detection sensitivity. This approach would be particularly favorable when the exact field profile is unknown or likely to be spatially non-uniform in both time and space. It can also be used to guide the user when deciding on which of the two earlier approaches should be best pursued.

\section{Conclusion}

Using the exact expression for a focused Gaussian beam, we conduct an examination of several parameters which affect the E-FISH signal, and its consequent use as a diagnostic for electric field measurements. One of the key findings of this work is that, apart from the magnitude of the externally applied electric field (the intended measurement quantity), both the length and shape of this field profile also have a distinct effect on the signal generation. This has profound consequences for effective implementation of the E-FISH diagnostic, since unlike other parameters such as the Rayleigh range, wave vector mismatch and non-linear susceptibility, the physical length and shape of the electric field profile are often challenging to determine in a plasma. Failure to account for this contribution can lead to erroneous interpretations of the signal, and thus an inaccurate electric field measurement. While similar effects of the interaction length, or equivalently, medium length, have been reported in THG microscopy, the interaction lengths relevant to E-FISH (when used for diagnosing gas plasmas), are generally much longer, similar to SHG microscopy performed in bulk non-centrosymmetric media. In summary, we urge caution 
when continuing to use the E-FISH diagnostic with a single co-propagating beam, and suggest several options for mitigating the issues raised in this work. These include increasing the Rayleigh range of the probe beam to ensure compliance with the plane-wave approximation, or utilizing two crossed laser beams to localize the measurement and eliminate any signal dependence on the spatial profile of the external electric field beyond the intersection volume.

\section{Acknowledgments}

The work was partially supported by the French General Directorate of Armaments (DGA) under the EP-DGA convention N2790, the French National Research Agency, ANR (ASPEN Project), LabEx Plas@Par and the French-Russian international laboratory LIA KaPPA 'Kinetics and Physics of Pulsed Plasmas and their Afterglow'. The authors would also like to thank Dr. Olivier Guaitella, Dr. Jean-Paul Booth and Dr. Cyril Drag for various equipment essential for conducting these experiments, and to Pascal Pariset for apparatus design \& mechanical work. Insightful discussions with Dr. Christophe Blondel, Dr. Yifei Zhu and Dr. Emmanuel Beaurepaire, as well as helpful contributions from Ms. Orel Inna, are also gratefully acknowledged.

\section{References}

1. Dogariu, A., Goldberg, B. M., O’Byrne, S., \& Miles, R. B. (2017). Species-independent femtosecond localized electric field measurement. Physical Review Applied, 7(2), 024024.

2. Goldberg, B. M., Chng, T. L., Dogariu, A., \& Miles, R. B. (2018). Electric field measurements in a near atmospheric pressure nanosecond pulse discharge with picosecond electric field induced second harmonic generation. Applied Physics Letters, 112(6), 064102.

3. Chng, T. L., Orel, I. S., Starikovskaia, S. M., \& Adamovich, I. V. (2019). Electric field induced second harmonic (E-FISH) generation for characterization of fast ionization wave discharges at moderate and low pressures. Plasma Sources Science and Technology, 28(4), 045004.

4. Huang, B., Zhang, C., Adamovich, I., Akishev, Y., \& Shao, T. (2020). Surface ionization wave propagation in the nanosecond pulsed surface dielectric barrier discharge: the influence of dielectric material and pulse repetition rate. Plasma Sources Science and Technology, 29(4), 044001.

5. Simeni, M. S., Tang, Y., Frederickson, K., \& Adamovich, I. V. (2018). Electric field distribution in a surface plasma flow actuator powered by ns discharge pulse trains. Plasma Sources Science and Technology, 27(10), 104001.

6. Orr, K., Tang, Y., Simeni, M. S., van den Bekerom, D., \& Adamovich, I. V. (2020). Measurements of electric field in an atmospheric pressure helium plasma jet by the E-FISH method. Plasma Sources Science and Technology, 29(3), 035019.

7. Cui, Y., Zhuang, C., \& Zeng, R. (2019). Electric field measurements under DC corona discharges in ambient air by electric field induced second harmonic generation. Applied Physics Letters, 115(24), 244101.

8. Goldberg, B. M., Reuter, S., Dogariu, A., \& Miles, R. B. (2019). 1D time evolving electric field profile measurements with sub-ns resolution using the E-FISH method. Optics 
letters, 44(15), 3853-3856.

9. Meehan, K. C., Starikovskiy, A., \& Miles, R. (2020). Two Component Electric Field Dynamics of a ns-SDBD Plasma with Sub-Nanosecond Resolution by Femtosecond EFISH. In AIAA Scitech 2020 Forum (p. 1747).

10. Chng, T. L., Naphade, M., Goldberg, B. M., Adamovich, I. V., \& Starikovskaia, S. M. (2020). Electric field vector measurements via nanosecond electric-field-induced second-harmonic generation. Optics Letters, 45(7), 1942-1945.

11. Chng, T. L., Brisset, A., Jeanney, P., Starikovskaia, S. M., Adamovich, I. V., \& Tardiveau, P. (2019). Electric field evolution in a diffuse ionization wave nanosecond pulse discharge in atmospheric pressure air. Plasma Sources Science and Technology, 28(9), 09LT02.

12. Penndorf, R. (1957). Tables of the refractive index for standard air and the Rayleigh scattering coefficient for the spectral region between 0.2 and $20.0 \mu$ and their application to atmospheric optics. Josa, 47(2), 176-182.

13. Boyd, R. W. (2019). Nonlinear optics, (Chapter 2.10.), Academic press Inc., United States.

14. Ward, J. F., \& New, G. H. C. (1969). Optical third harmonic generation in gases by a focused laser beam. Physical Review, 185(1), 57.

15. Feng, S., \& Winful, H. G. (2001). Physical origin of the Gouy phase shift. Optics letters, 26(8), 485-487.

16. Boyd, G. D., \& Kleinman, D. A. (1968). Parametric interaction of focused Gaussian light beams. Journal of Applied Physics, 39(8), 3597-3639.

17. Bjorklund, G. C. (1975). Effects of focusing on third-order nonlinear processes in isotropic media. IEEE Journal of Quantum Electronics, 11(6), 287-296.

18. van der Schans, M. (2018). Experiments on the physics of pulsed plasma jets. PhD thesis, Technische Universiteit Eindhoven, Department of Applied Physics, Proefschrift.

19. Bigio, I. J., Finn, R. S., \& Ward, J. F. (1975). Electric-field induced harmonic generation as a probe of the focal region of a laser beam. Applied optics, 14(2), 336-342.

20. Bentaleb, S., Blin-Simiand, N., Jeanney, P., Magne, L., Moreau, N., Pasquiers, S., \& Tardiveau, P. (2015). Ignition of lean air/hydrocarbon mixtures at low temperature by a single corona discharge nanosecond pulse. AerospaceLab, 10.

21. Shcherbanev, S. A., Ding, C., Starikovskaia, S. M., \& Popov, N. A. (2019). Filamentary nanosecond surface dielectric barrier discharge. Plasma properties in the filaments. Plasma Sources Science and Technology, 28(6), 065013.

22. Moreaux, L., Sandre, O., Charpak, S., Blanchard-Desce, M., \& Mertz, J. (2001). Coherent scattering in multi-harmonic light microscopy. Biophys. J., 80, 1568-1574.

23. Cheng, J. X., \& Xie, X. S. (2002). Green's function formulation for third-harmonic generation microscopy. J. Opt. Soc. Am. B, 19(7), 1604-1610.

24. Stoller, P., Celliers, P. M., Reiser, K. M., \& Rubenchik, A. M. (2003). Quantitative secondharmonic generation microscopy in collagen. Appl. Opt., 42(25), 5209-5219.

25. D. Débarre, W. Suppato, A. M. Pena, A. Fabre, T. Tordjmann, L. Combettes, M. C. SchanneKlein, and E. Beaurepaire (2006). Imaging lipid bodies in cells and tissues using thirdharmonic generation microscopy. Nat. Methods, 3 (1), 47 - 53. 
26. Erikson, A., Örtegren, J., Hompland, T., Davies, C. d. L., \& Lindgren, M. (2007). Quantification of the second-order nonlinear susceptibility of collagen I using a laser scanning microscope. J. Biomed. Optics, 12(4), 044002.

27. Sandkuijl, D., Tuer, A. E., Tokarz, D., Sipe, J. E., \& Barzda, V. (2013). Numerical secondand third-harmonic generation microscopy. J. Opt. Soc. Am. B, 30(2), 382-395.

28. S. Bancelin, C. Aimé, I. Gusachenko, L. Kowalczuk, G. Latour, T. Coradin, and M.-C. Schanne-Klein, (2014). Determination of collagen fibril size via absolute measurements of second-harmonic generation signals. Nat. Commun., 5, 4920.

29. Levy, U., \& Silberberg, Y. (2015). Second and third harmonic waves excited by focused Gaussian beams. Optics express, 23(21), 27795-27805.

30. Campagnola, P. J., Millard, A. C., Terasaki, M., Hoppe, P. E., Malone, C. J., \& Mohler, W. A. (2002). Three-dimensional high-resolution second-harmonic generation imaging of endogenous structural proteins in biological tissues. Biophysical journal, 82(1), 493-508.

31. Zipfel, W. R., Williams, R. M., Christie, R., Nikitin, A. Y., Hyman, B. T., \& Webb, W. W. (2003). Live tissue intrinsic emission microscopy using multiphoton-excited native fluorescence and second harmonic generation. Proceedings of the National Academy of Sciences, 100(12), 7075-7080.. 